\title{
Are VEGFR-TKIs effective or safe for patients with advanced non-small cell lung cancer?
}

\author{
Shuai Wang ${ }^{1}$, Zhe Yang ${ }^{2}$ and Zhou Wang ${ }^{1}$ \\ ${ }^{1}$ Department of Thoracic Surgery, Provincial Hospital Affiliated to Shandong University, Jinan, Shandong, People's Republic \\ of China \\ 2 Department of Oncology, Provincial Hospital Affiliated to Shandong University, Jinan, Shandong, People's Republic of China \\ Correspondence to: Zhou Wang, email: wz620226@hotmail.com \\ Keywords: lung cancer, angiogenesis inhibitors, VEGFR, TKIs, meta analysis
}

Received: March 14, $2015 \quad$ Accepted: June 05, $2015 \quad$ Published: June 19, 2015

This is an open-access article distributed under the terms of the Creative Commons Attribution License, which permits unrestricted use, distribution, and reproduction in any medium, provided the original author and source are credited.

\section{ABSTRACT}

Vascular endothelial growth factor receptor tyrosine kinase inhibitors (VEGFRTKIs) might be new therapeutic strategies for advanced non-small cell lung cancer (NSCLC). Here a total of 12,520 patients from 23 randomized controlled trials (RCTs) were enrolled to evaluate the efficacy and safety of VEGFR-TKIs quantitatively in advanced NSCLC. Compared with non-VEGFR-TKIs, VEGFR-TKIs regimen significantly improved progression-free survival (PFS) [hazard ratio (HR): $0.839,95 \%$ confidence interval (CI): 0.805-0.874, $P<0.001$ ], objective response rates (ORR) [relative risk (RR): 1.374, 95\% CI: 1.193-1.583, $P<0.001$ ] and disease control rates (DCR) (RR: 1.113, 95\% CI: 1.027-1.206, $P=0.009$ ), but not overall survival (OS) (HR: 0.960, 95\% CI: $0.921-1.002, P=0.060$ ) for NSCLC patients. The RR of all-grade neutropenia, thrombocytopenia, hypertension, hemorrhage, fatigue, anorexia, stomatitis, diarrhea, rash, hand-foot skin reaction (HFSR) were increased in patients received VEGFRTKIs. As for high-grade ( $\geq 3$ ) adverse events (AEs), VEGFR-TKIs were associated with higher RR of neutropenia, thrombocytopenia, hypertension, fatigue, stomatitis, diarrhea, rash and HFSR. This study demonstrates VEGFR-TKIs improve PFS, ORR and DCR, but not OS in advanced NSCLC patients. VEGFR-TKIs induce more frequent and serious AEs compared with control therapies.

\section{INTRODUCTION}

Standard therapy for advanced NSCLC is platinumbased palliative chemotherapy. Although $30-50 \%$ of NSCLC patients respond to platinum-based regimens, the median over survival (OS) is still 8-12 months [1,2]. Traditional chemotherapy has limited clinical benefits. The "one size fits all" treatment model must be changed. The effective and less toxic drugs are urgently needed.

Over the past decade, progress of NSCLC biology promoted the development of targeted agents. Those targeted agents specifically inhibit vital signaling pathways, including epidermal growth factor receptor (EGFR) and vascular endothelial growth factor recepter (VEGFR) [3]. Currently, targeted therapies have emerged as novel therapeutic options and changed the treatment paradigm of NSCLC. VEGFR, a critical pathway in tumor progression, represents an important target in NSCLC
[4]. There are two major categories of agents targeting VEGFR pathway: VEGF antibodies and VEGFR tyrosine kinase inhibitors (VEGFR-TKIs).

Several VEGFR-TKIs have been developed for targeted therapies in NSCLC, such as sorafenib, sunitinib, cediranib and vandetanib. The clinical efficacy of VEGFR-TKIs in advanced NSCLC, as a part of combination therapies or single agent had been evaluated [5-10]. But the results were inconsistent. A previous meta-analysis showed that chemotherapy plus VEGFRTKIs significantly improved the progression-free survival (PFS), objective response rates (ORR) and disease control rates (DCR), but not overall survival (OS) [11]. Another meta-analysis suggested that VEGFR-TKIs significantly increased risk of death compared with non-VEGFRTKIs [12]. In a recent meta-analysis, Shaodong $\mathrm{H}$ et al demonstrated that angiogenesis inhibitors had significant advantages over non-angiogenesis inhibitors in terms 
of PFS, OS, ORR and DCR in advanced NSCLC [13]. However, the meta analysis included VEGF antibodybased agents (bevacizumab, aflibercept and ramucirumab). Since then, several novel randomized controlled trials (RCTs) are emerging. Furthermore, previous studies showed different and even contradictory conclusions. The overall efficacy and safety of VEGFR-TKIs are undetermined. A more comprehensive review of previous studies is needed. In this study, we performed a pooled analysis of currently published RCTs to summarize the up- to-date evidence.

\section{RESULTS}

\section{Literature search and study characteristics}

A total of 517 articles were retrieved from initial electronic database and meeting abstracts. The selection steps in different phases were summarized in Figure 1.

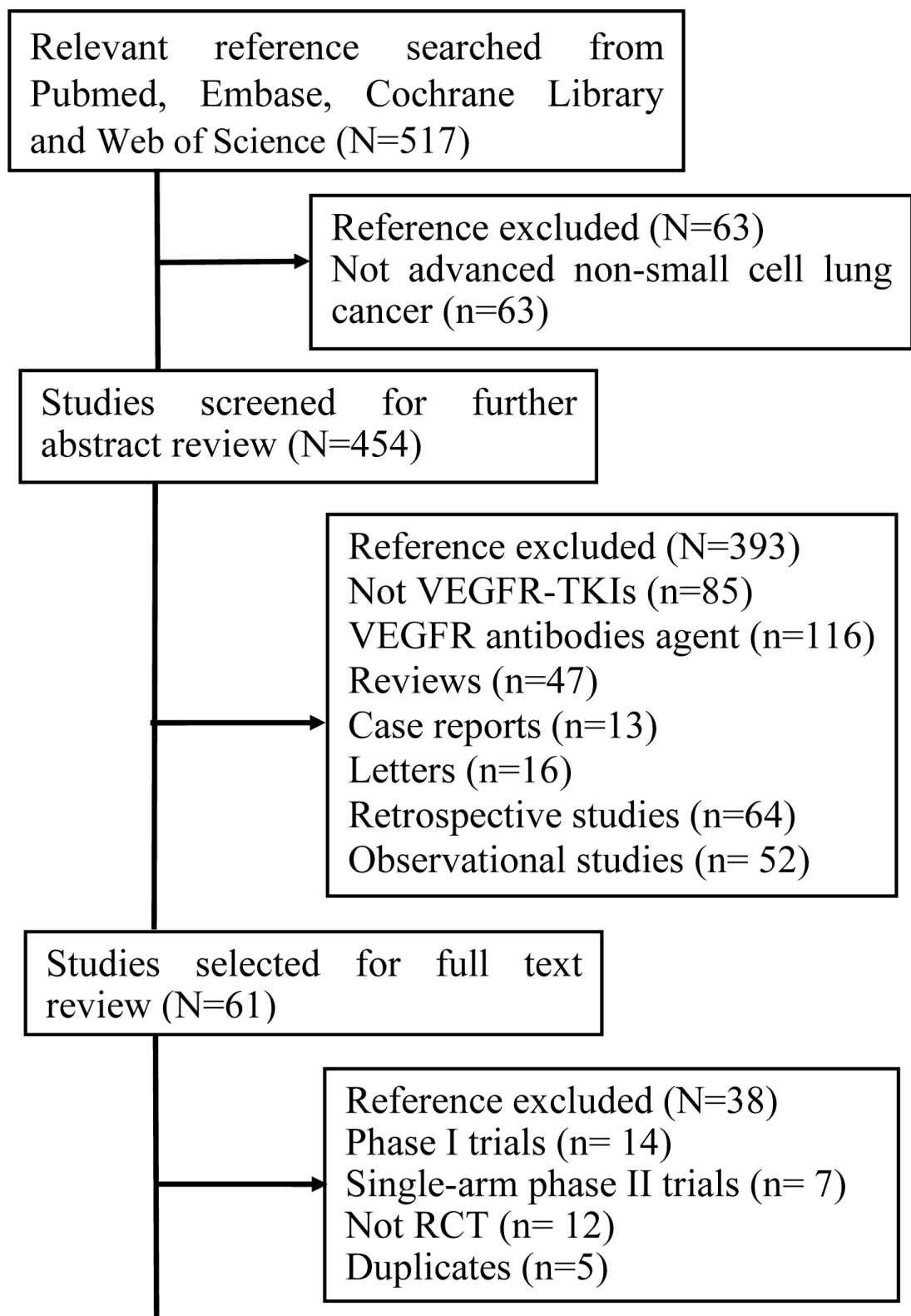

23 studies of full publications in English were included in meta-analysis

Figure 1: Study flow chart showing the process for selecting eligible publications. N: the number of studies; RCT: randomized controlled trial. 
Table 1: Characteristics of studies included in the meta-analysis

\begin{tabular}{|c|c|c|c|c|c|c|c|c|c|c|c|c|c|}
\hline Study & Year & $\begin{array}{l}\text { Trial } \\
\text { phase }\end{array}$ & Line & Arms & Cases & $\begin{array}{l}\text { Stage } \\
\text { IIIB/IV } \\
(\mathrm{n} / \mathrm{n})\end{array}$ & $\begin{array}{l}\text { Median } \\
\text { TT } \\
\text { (months) }\end{array}$ & $\begin{array}{l}\text { Median } \\
\text { age } \\
\text { (years) }\end{array}$ & $\begin{array}{l}\text { Objective } \\
\text { response } \\
\text { rate }(n / N)\end{array}$ & $\begin{array}{l}\text { Disease } \\
\text { control } \\
\text { rate }\end{array}$ & $\begin{array}{l}\text { Median } \\
\text { PFS } \\
\text { (months) }\end{array}$ & $\begin{array}{l}\text { Median } \\
\text { OS } \\
\text { (months) }\end{array}$ & $\begin{array}{l}\text { Jadad } \\
\text { score }\end{array}$ \\
\hline Laurie & 2014 & 3 & 1 & Cedi + PC & 153 & $14 / 139$ & 3.5 & 63 & $74 / 143$ & NA & 5.5 & 12.2 & 4 \\
\hline [14] & & & & Plac + PC & 153 & $7 / 146$ & 2.8 & 62 & $49 / 144$ & & 5.5 & 12.1 & \\
\hline Dy & 2013 & 2 & 1 & Cedi + GC & 58 & NA & 2.5 & 65 & $11 / 58$ & NA & 6.3 & 12 & 3 \\
\hline [15] & & & & GC & 29 & & 2.8 & 64 & $6 / 29$ & & 4.5 & 9.9 & \\
\hline Goss & 2010 & 2 & 1 & Cedi + PC & 126 & NA & 4.7 & 60 & $48 / 126$ & NA & 5.6 & NA & 3 \\
\hline [16] & & & & $\mathrm{Plac}+\mathrm{PC}$ & 125 & & 4.4 & 58 & $20 / 125$ & & 5.0 & & \\
\hline Scagliotti & $2012 b$ & 3 & 1 & Mote + PC & 956 & $73 / 468$ & 4.1 & 60 & $382 / 956$ & $774 / 956$ & 5.6 & 13 & 4 \\
\hline [10] & & & & $\mathrm{Plac}+\mathrm{PC}$ & 949 & $79 / 470$ & 4.1 & 60 & $247 / 949$ & $702 / 949$ & 5.4 & 11 & \\
\hline Reck & 2014 & 3 & 2 & Nint + Doc & 655 & $148 / 399^{1}$ & 3.4 & 60 & $29 / 655$ & $354 / 655$ & 3.5 & 10.1 & 5 \\
\hline [17] & & & & Plac + Doc & 659 & $146 / 408$ & 2.8 & 60 & $22 / 659$ & $272 / 659$ & 2.7 & 9.1 & \\
\hline Hanna & 2013 & 3 & 2 & Nint + Pem & 353 & NA & NA & 60 & NA & NA & 4.4 & 12.2 & 4 \\
\hline [18] & & & & Plac + Pem & 360 & & & 59 & & & 3.6 & 12.7 & \\
\hline Scagliotti & 2013 & 2 & 1 & Pazo + Pem & 62 & $2 / 59^{2}$ & 3.6 & 62 & $14 / 62$ & $27 / 62$ & 5.8 & NA & 2 \\
\hline [19] & & & & $\mathrm{DDP}+\mathrm{Pem}$ & 35 & $3 / 32$ & 2.7 & 64 & $12 / 35$ & $26 / 35$ & 5.3 & & \\
\hline Wakelee & 2012 & 2 & $>2$ & Sora & 50 & NA & 2.0 & 64.5 & NA & $27 / 50$ & 3.3 & 13.7 & 5 \\
\hline [20] & & & & Plac & 31 & & 2.0 & 69 & & $7 / 31$ & 2 & 9 & \\
\hline Paz-Ares & 2012 & 3 & 1 & Sora + GP & 385 & $47 / 338$ & 1.8 & 60 & $107 / 385$ & $239 / 385$ & 6 & 12.4 & 5 \\
\hline [21] & & & & $\mathrm{Plac}+\mathrm{GP}$ & 387 & $47 / 340$ & 2.0 & 58 & $100 / 340$ & $244 / 340$ & 5.5 & 12.5 & \\
\hline Spigel & 2011 & 2 & $\geq 2$ & Sora + Erlo & 111 & NA & 3.3 & 65 & $9 / 111$ & $60 / 111$ & 3.38 & 7.62 & 4 \\
\hline [22] & & & & Plac + Erlo & 55 & & 3.3 & 65 & $6 / 111$ & $21 / 111$ & 1.94 & 7.23 & \\
\hline Scagliotti & 2010 & 3 & 1 & Sora + PC & 464 & $44 / 420$ & 3.9 & 62 & $127 / 464$ & $232 / 464$ & 4.6 & 10.7 & 4 \\
\hline [9] & & & & $\mathrm{Plac}+\mathrm{PC}$ & 462 & $47 / 415$ & 4.2 & 63 & $111 / 462$ & $259 / 464$ & 5.4 & 10.6 & \\
\hline Heist & 2014 & 2 & $\geq 2$ & Suni + Pem & 41 & $3 / 38$ & 4.2 & 63 & $9 / 41$ & $30 / 41$ & 3.7 & 6.7 & 3 \\
\hline [23] & & & & Pem & 42 & $5 / 37$ & 4.2 & 63 & $6 / 42$ & $27 / 42$ & 4.9 & 10.5 & \\
\hline
\end{tabular}




\begin{tabular}{|c|c|c|c|c|c|c|c|c|c|c|c|c|c|}
\hline Study & Year & $\begin{array}{l}\text { Trial } \\
\text { phase }\end{array}$ & Line & Arms & Cases & $\begin{array}{l}\text { Stage } \\
\text { IIIB/IV } \\
(\mathrm{n} / \mathrm{n})\end{array}$ & $\begin{array}{l}\text { Median } \\
\text { TT } \\
\text { (months) }\end{array}$ & $\begin{array}{l}\text { Median } \\
\text { age } \\
\text { (years) }\end{array}$ & $\begin{array}{l}\text { Objective } \\
\text { response } \\
\text { rate }(n / N)\end{array}$ & $\begin{array}{l}\text { Disease } \\
\text { control } \\
\text { rate }\end{array}$ & $\begin{array}{l}\text { Median } \\
\text { PFS } \\
\text { (months) }\end{array}$ & $\begin{array}{l}\text { Median } \\
\text { OS } \\
\text { (months) }\end{array}$ & $\begin{array}{l}\text { Jadad } \\
\text { score }\end{array}$ \\
\hline Groen & 2013 & 2 & 2 or & Suni + Erlo & 65 & $1 / 63^{3}$ & 2.0 & 59 & $3 / 65$ & NA & 2.8 & 8.2 & 5 \\
\hline [24] & & & 3 & Plac + Erlo & 67 & $0 / 67$ & 2.8 & 61 & $2 / 67$ & & 2.0 & 7.6 & \\
\hline Scagliotti & $2012 a$ & 3 & $\geq 2$ & Suni + Erlo & 480 & $42 / 438$ & 4.3 & 61 & $51 / 480$ & $206 / 480$ & 3.6 & 9.0 & 5 \\
\hline$[25]$ & & & & Plac + Erlo & 480 & $32 / 448$ & 4.4 & 61 & $33 / 480$ & $168 / 480$ & 2.0 & 8.5 & \\
\hline Aisner & 2013 & 2 & M & Vand & 80 & $5 / 68^{4}$ & 2.8 & 63.5 & $15 / 80$ & NA & 4.5 & 9.8 & 5 \\
\hline$[26]$ & & & & Plac & 82 & $9 / 66$ & 1.8 & 63 & $15 / 82$ & & 4.2 & 9.4 & \\
\hline Ahna & 2013 & 2 & M & Vand & 75 & $15 / 60$ & 2.0 & 61 & $14 / 75$ & NA & 2.7 & 15.6 & 4 \\
\hline [27] & & & & Plac & 42 & $12 / 30$ & 1.8 & 60.5 & $1 / 42$ & & 1.7 & 20.8 & \\
\hline Lee & 2012 & 3 & 2 or & Vand & 617 & $47 / 569$ & 3.4 & 60 & $16 / 617$ & NA & 1.9 & 8.5 & 3 \\
\hline [28] & & & 3 & Plac & 307 & $17 / 289$ & 2.5 & 60 & $2 / 307$ & & 1.8 & 7.8 & \\
\hline Natale & 2011 & 3 & 2 or & Vand & 623 & $106 / 517$ & 2.1 & 61 & $75 / 623$ & $254 / 623$ & 2.6 & 6.9 & 3 \\
\hline [29] & & & 3 & Erlo & 617 & $98 / 519$ & 2.0 & 61 & $74 / 617$ & $234 / 617$ & 2.0 & 7.8 & \\
\hline de Boer & 2011 & 3 & $\geq 2$ & Vand + Pem & 256 & $37 / 219$ & 3.4 & 60 & $49 / 256$ & $146 / 256$ & 4.1 & 10.5 & 4 \\
\hline [5] & & & & $\mathrm{Plac}+\mathrm{Pem}$ & 278 & $46 / 232$ & 2.8 & 60 & $22 / 278$ & $128 / 278$ & 2.8 & 9.2 & \\
\hline Herbst & 2010 & 3 & $\geq 2$ & Vand + Doc & 694 & $95 / 598^{5}$ & 2.8 & 59 & $120 / 694$ & $413 / 694$ & 4.0 & 10.6 & 5 \\
\hline [6] & & & & Plac + Doc & 697 & $106 / 590$ & 3.0 & 59 & $71 / 697$ & $380 / 697$ & 3.2 & 10.0 & \\
\hline Natale & 2009 & 2 & $\geq 2$ & Vand & 83 & $14 / 69$ & NA & 61 & $7 / 83$ & $37 / 69$ & 2.6 & NA & 3 \\
\hline$[30]$ & & & & Gefi & 85 & $22 / 63$ & & 63 & $1 / 63$ & $29 / 85$ & 1.9 & & \\
\hline Heymach & 2008 & 2 & 1 & Vand + PC & 56 & $7 / 49$ & NA & 60 & $18 / 56$ & NA & 6.0 & 10.2 & 3 \\
\hline [7] & & & & $\mathrm{PC}$ & 52 & $5 / 47$ & & 59 & $13 / 52$ & & 5.8 & 12.6 & \\
\hline Heymach & 2007 & 2 & 2 & Vand + Doc & 44 & $9 / 35$ & NA & 60 & $8 / 44$ & $28 / 44$ & 4.0 & 7.9 & 3 \\
\hline [8] & & & & $\mathrm{Plac}+\mathrm{Doc}$ & 41 & $13 / 28$ & & 58 & $5 / 41$ & $23 / 41$ & 2.8 & 13.4 & \\
\hline
\end{tabular}

1: 105 patients with stage $<$ IIIB in each arm and 3 patients missing the information; $2: 1$ patient missing the information; $3: 0 n e$ subject in the sunitinib arm with no informed consent who was randomized in error; 4:7 patients with recurrence disease in each arm; 5: not recorded for one patient in each group; 6: Data not available for one patient in the vandetanib+ docetaxel arm. TT: treatment time; Cedi: cediranib; Plac: Placebo; Mote: motesanib; Nint: nintedanib; Pazo: pazopanib; Sora: sorafenib; Suni: sunitinib; Vand: vandetanib; GC: gemcitabine + carboplatin; DC: docetaxel + carboplatin; Doc: docetaxel; GP gemcitabine + cisplatin; Erlo: erlotinib; PC: paclitaxel + carboplatin; DDP:cisplatin; Pem: pemetrexed; Gefi: gefitinib; NA: not available; M: maintenance therapy; PFS: progression-free survival; OS: overall survival. 
Finally, only 23 RCTs were included in our study. These RCTs enrolled a total of 12,520 patients (VEGFR-TKIs arm: 6487, control arm:6033). Among these 23 studies, 3 were on cediranib [14-16]; 1 on motesanib [10]; 2 on nintedanib $[17,18] ; 1$ on pazopanib [19]; 4 on sorafenib $[9,20-22] ; 3$ on sunitinib [23-25] and 9 on vandetanib [5-8, 26-30]. These RCTs comprised 12 phase II and 11 phase III clinical trials. Fourteen studies were published in recent three years. Eight trials were performed in first-line settings, 13 in $\geq$ second-line settings and 2 in maintenance. VEGFR-TKIs were compared with placebo in 6 trials and 17 trials were chemotherapy plus VEGFR-TKIs versus chemotherapy alone. The overall study quality was fair with a median Jadad score of 4, suggesting the quality of all RCTs was quite good. The characteristics of the 23
RCTs were listed in Table 1.

\section{Progression free survival}

Twenty three studies reported the PFS of 6,487 patients in VEGFR-TKIs arm and 6,033 patients in control arm. Heterogeneity analysis revealed that there was no significant between-study heterogeneity (chi-squared $=$ 31.88 , d.f. $=22, P=0.079$, I-squared $=31.0 \%$ ). A metaanalysis was therefore carried out using the fixed-effects model. A statistically significant improvement in PFS was observed favoring VEGFR-TKIs groups [hazard ratio (HR): $0.839,95 \%$ confident intervals (CI): 0.805-0.874, $P$ $<0.001$ ) (Figure 2A).

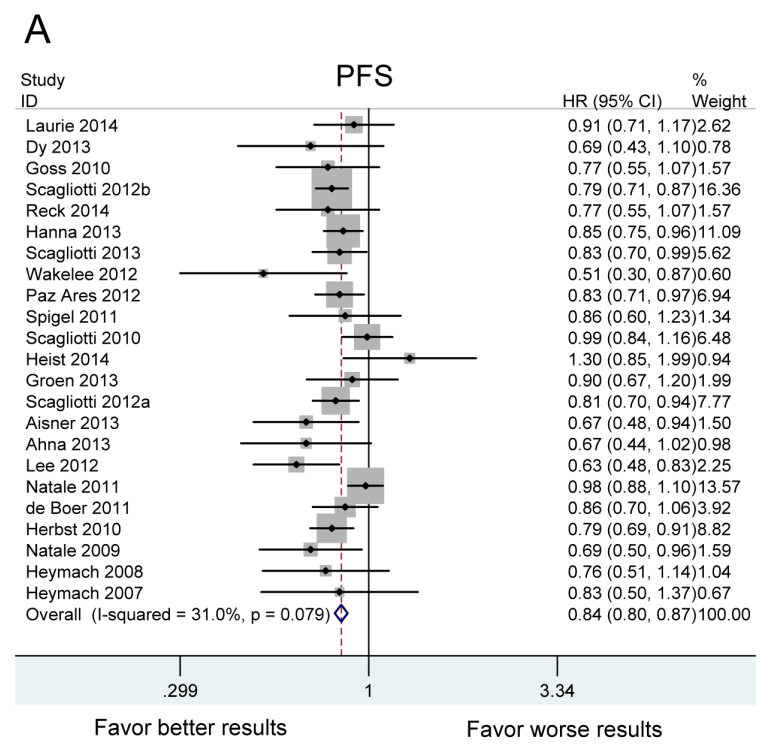

B
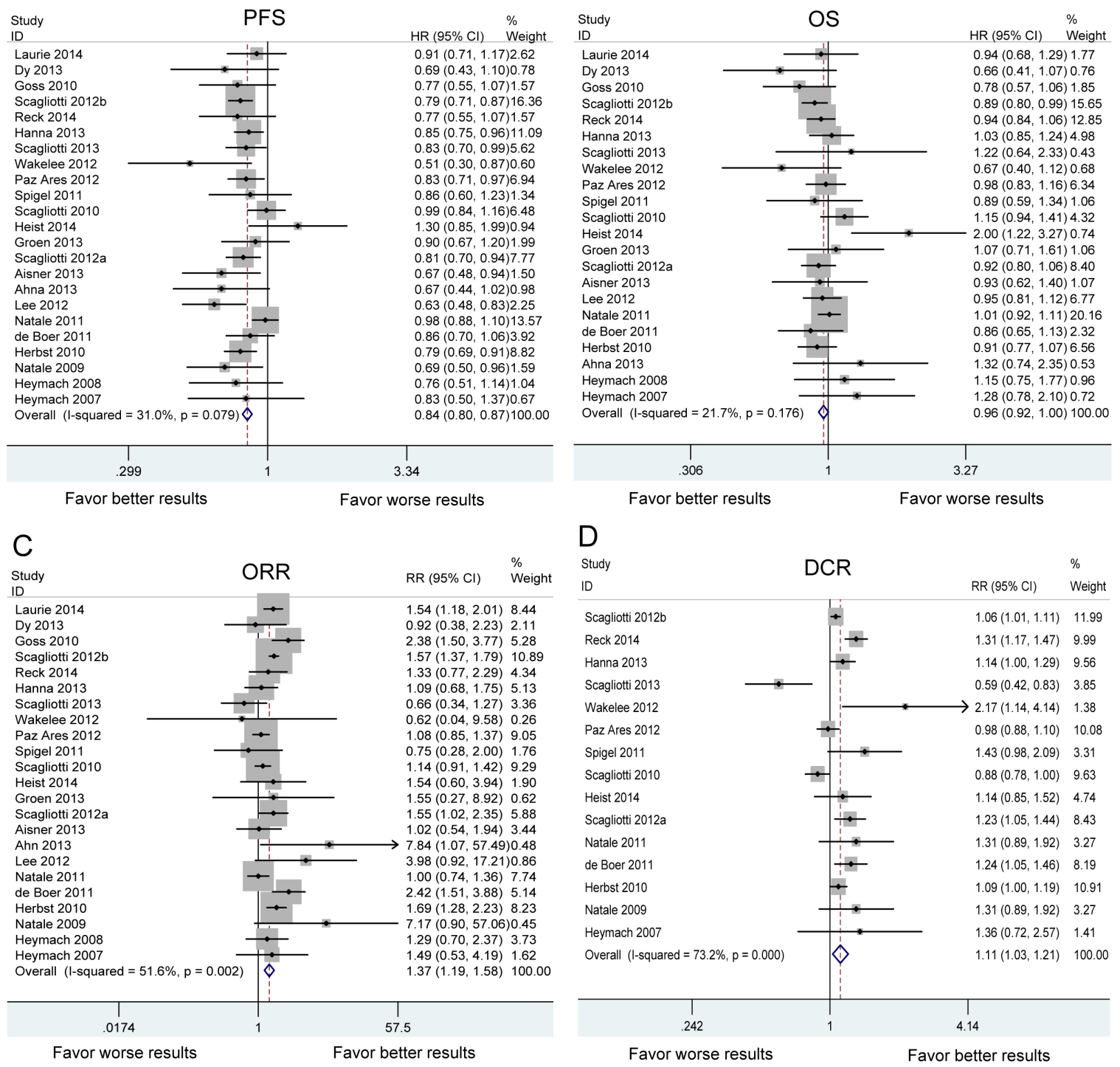

Figure 2: The pooled analysis of progression-free survival (PFS) A. overall survival (OS) B. objective response rate (ORR) C. and disease control rate (DCR) D. in NSCLC patients who received VEGFR-TKI therapies compared to control therapies. HR: hazard ratio. RR: relative risk. Squares indicate study-specific HR or RR (size of the square reflects the study-specific statistical weight); horizontal lines indicate $95 \%$ confidence interval (CI); diamond indicates the summary HR or RR estimate with its $95 \%$ CI. 
Subgroups analyses were performed based on the individual VEGFR-TKI, treatment line and treatment regimen (Table 2). As shown in Figure 3A, significant
PFS benefit was found in all VEGFR-TKIs. VEGFRTKIs improved the PFS in first-line, $\geq$ second-line and maintenance treatment (Figure 4A). A statistically

\section{A}

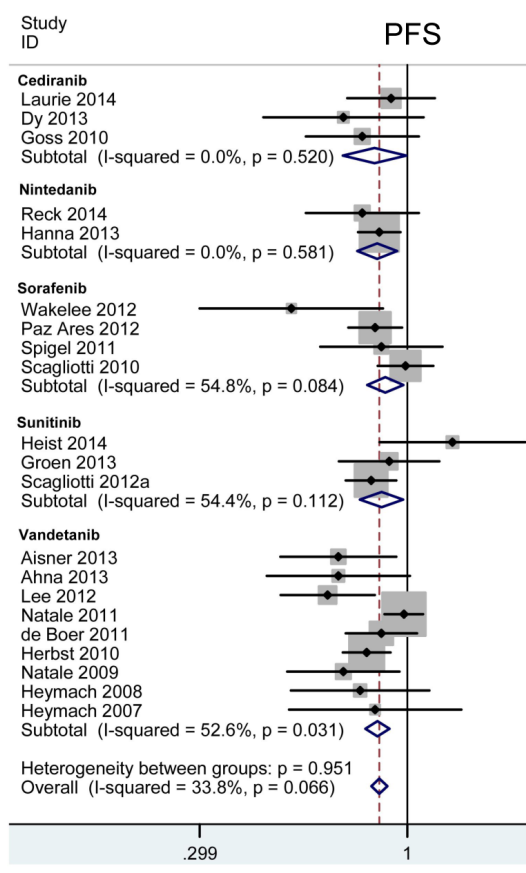

Favor better results

C

Study

\section{Cediranib}

Cediranib

Dy 2013

Subtotal (I-squared $=54.1 \%, p=0.113$ )

Nintedanib

Reck 2014
Hanna 2013

Subtotal (I-squared $=0.0 \%, p=0.588$ )

Sorafenib

Wakelee 2012

Paz Ares 2012

Spigel 2011

Subtotal ( $($-squared $=0.0 \%, p=0.832$ )

Sunitinib

Heist 2014

Groen 2013

Scagliotti $2012 a$
Subtotal $(I-$ squared $=0.0 \%, p=1.000)$

Vandetanib

Aisner 2013

Ahn 2013

Lee 2012

Natale 2011
de Boer 2011

Herbst 2010
Natale 2009

Natale 2009

Heymach 2008

Subtotal (l-squared $=59.1 \%, p=0.012$ )

Heterogeneity between groups: $p=0.02$

Overall (I-squared $=45.3 \%, p=0.013$ )

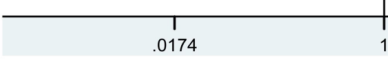

Favor worse results
ORR ES $(95 \% \mathrm{Cl}) \quad$ Weight

$1.54(1.18,2.01) \quad 12.83$

$0.92(0.38,2.23) \quad 1.16$

$\begin{array}{ll}2.38(1.50,3.77) & 4.28 \\ 1.65(1.32,2.06) & 18.28\end{array}$

$1.33(0.77,2.29) \quad 3.09$ $\begin{array}{ll}1.309(0.68,1.75) & 4.07 \\ 1.19(0.83,1.70) & 7.16\end{array}$

$0.62(0.04,9.58) \quad 0.12$ $\begin{array}{ll}1.08(0.85,1.37) & 16.47 \\ 0.75(0.28,2.00) & 0.94\end{array}$ $\begin{array}{ll}0.14(0.28,2.00) & 0.94 \\ 1.14(0.91,1.42) & 18.38\end{array}$ $\begin{array}{ll}1.10(0.94,1.29) & 35.92\end{array}$

$\begin{array}{ll}1.54(0.60,3.94) & 1.03 \\ 1.55(0.27,8.92) & 0.30\end{array}$ $\begin{array}{ll}1.55(0.27,8.92) & 0.30 \\ 1.55(1.02,2.35) & 5.22\end{array}$ $\begin{array}{ll}1.55(1.02,2.35) & 5.22 \\ 1.55(1.07,2.25) & 6.55\end{array}$ $\begin{array}{ll}1.02(0.54,1.94) & 2.19 \\ 7.84(1.07,57.49) & 0.23 \\ 3 & \end{array}$ $\begin{array}{ll}3.98(0.92,17.21) & 0.42 \\ 1.00(0.74,1.36) & 9.83\end{array}$ $2.42(1.51,3.88) \quad 4.09$ $\begin{array}{ll}1.69(1.28,2.23) & 11.81 \\ 7.17(0.90,57.06) & 0.21\end{array}$ $\begin{array}{ll}1.29(0.70,2.37) & 0.21 \\ 1.46\end{array}$ $\begin{array}{ll}.49(0.53,4.19) & 0.85 \\ .47(1.24,1.74) & 32.09\end{array}$

$1.34(1.21,1.47) \quad 100.00$

Figure 3: Subgroup analysis based on individual VEGFR-TKI in advanced NSCLC patients in terms of progressionfree survival (PFS) A. overall survival (OS) B. objective response rate (ORR) C. and disease control rate (DCR). D. Squares indicate study-specific HR or RR (size of the square reflects the study-specific statistical weight); horizontal lines indicate $95 \%$ confidence interval (CI); diamond indicates the summary HR or RR estimate with its $95 \%$ CI. 
Table 2: Results of subgroup analysis according to drug Class, treatment line and regimens for non-small cell lung cancer

\begin{tabular}{|c|c|c|c|c|c|c|c|c|c|c|c|}
\hline \multicolumn{3}{|c|}{ Stratified analysis } & \multirow{2}{*}{$\begin{array}{l}\text { No. of } \\
\text { studies }\end{array}$} & \multirow{2}{*}{$\begin{array}{l}\text { Cases (TKIs } \\
\text { /control arm) }\end{array}$} & \multicolumn{2}{|c|}{ Heterogeneity } & \multirow{2}{*}{$\begin{array}{l}\text { Pooled HR/RR } \\
(95 \% \mathrm{CI})\end{array}$} & \multicolumn{2}{|c|}{ Begg's Test } & \multirow{2}{*}{$\begin{array}{l}\text { Egger's test } \\
\text { P value }\end{array}$} & \multirow{2}{*}{$\begin{array}{l}\text { Metaregression } \\
\text { P value }\end{array}$} \\
\hline & & & & & $\mathrm{I}^{2}(\%)$ & $\mathrm{P}$ value & & $\bar{Z}$ & $\mathrm{P}$ value & & \\
\hline \multirow[t]{10}{*}{ PFS } & TKIs & Cediranib & 3 & $337 / 307$ & 0.0 & 0.520 & $0.827(0.687-0.994)$ & 1.04 & 0.296 & 0.191 & 0.819 \\
\hline & & Nintedanib & 2 & $1008 / 1019$ & 0.0 & 0.581 & $0.840(0.748-0.943)$ & 0.0 & 1.000 & - & \\
\hline & & Sorafenib & 4 & $1010 / 935$ & 35.5 & 0.212 & $0.880(0.792-0.977)$ & 0.34 & 0.734 & 0.334 & \\
\hline & & Sunitinib & 3 & $586 / 589$ & 54.4 & 0.112 & $0.861(0.759-0.976)$ & 1.04 & 0.296 & 0.231 & \\
\hline & & Vandetanib & 9 & $2528 / 2199$ & 52.6 & 0.031 & $0.786(0.697-0.887)$ & 0.31 & 0.754 & 0.056 & \\
\hline & Line & $1 \mathrm{st}$ & 8 & $2260 / 2192$ & 0.0 & 0.433 & $0.834(0.783-0.889)$ & 0.37 & 0.711 & 0.899 & 0.416 \\
\hline & & $\geq 2$ nd & 13 & $4072 / 3717$ & 45.0 & 0.040 & $0.831(0.764-0.905)$ & 0.33 & 0.743 & 0.244 & \\
\hline & & Maintenance & 2 & $155 / 124$ & 0.0 & 1.000 & $0.670(0.516-0.870)$ & 0.00 & 1.000 & - & \\
\hline & Regimen & Combination & 17 & $4959 / 4869$ & 0.0 & 0.701 & $0.835(0.798-0.875)$ & 0.49 & 0.621 & 0.442 & 0.261 \\
\hline & & Monotherapy & 6 & $1528 / 1164$ & 73.9 & 0.002 & $0.707(0.560-0.892)$ & 0.38 & 0.707 & 0.054 & \\
\hline \multirow[t]{10}{*}{ OS } & TKIs & Cediranib & 3 & $337 / 307$ & 0.0 & 0.453 & $0.817(0.668-1.000)$ & 0.0 & 1.000 & 0.484 & 0.322 \\
\hline & & Nintedanib & 2 & $1008 / 1019$ & 0.0 & 0.420 & $0.964(0.873-1.066)$ & 0.0 & 1.000 & - & \\
\hline & & Sorafenib & 4 & $1010 / 935$ & 34.0 & 0.208 & $0.988(0.838-1.166)$ & 1.02 & 0.308 & 0.315 & \\
\hline & & Sunitinib & 3 & $586 / 589$ & 77.8 & 0.011 & $1.193(0.784-1.813)$ & 1.04 & 0.296 & 0.348 & \\
\hline & & Vandetanib & 8 & $2445 / 2114$ & 0.0 & 0.671 & $0.981(0.917-1.050)$ & 1.36 & 0.174 & 0.537 & \\
\hline & Line & $1 \mathrm{st}$ & 8 & $2260 / 2192$ & 29.7 & 0.191 & $0.939(0.872-1.012)$ & 0.12 & 0.902 & 0.763 & 0.271 \\
\hline & & $\geq 2$ nd & 12 & $3989 / 3632$ & 27.8 & 0.172 & $0.969(0.920-1.020)$ & -0.07 & 1.000 & 0.602 & \\
\hline & & Maintenance & 2 & $155 / 124$ & 0.0 & 0.332 & $1.045(0.749-1.457)$ & 0.00 & 1.000 & - & \\
\hline & Regimen & Combination & 17 & $4959 / 4869$ & 28.6 & 0.131 & $0.949(0.903-0.998)$ & 1.61 & 0.108 & 0.158 & 0.227 \\
\hline & & Monotherapy & 5 & $1445 / 1079$ & 0.0 & 0.446 & $0.988(0.914-1.068)$ & -0.24 & 1.000 & 0.602 & \\
\hline \multirow{2}{*}{\multicolumn{3}{|c|}{ Stratified analysis }} & \multirow{2}{*}{$\begin{array}{l}\text { No. of } \\
\text { studies }\end{array}$} & \multirow{2}{*}{$\begin{array}{l}\text { Cases (TKIs } \\
\text { /control arm) }\end{array}$} & \multicolumn{2}{|c|}{ Heterogeneity } & \multirow{2}{*}{$\begin{array}{l}\text { Pooled HR/RR } \\
-(95 \% \mathrm{CI})\end{array}$} & \multicolumn{2}{|c|}{ Begg's Test } & Egger's test & Metaregression \\
\hline & & & & & $\overline{I^{2}(\%)}$ & P value & & $\bar{Z}$ & $\mathrm{P}$ value & - $P$ value & $P$ value \\
\hline \multirow[t]{10}{*}{ ORR } & TKIs & Cediranib & 3 & $337 / 307$ & 54.1 & 0.113 & $1.652(1.101-2.479)$ & 0.0 & 1.000 & 0.895 & 0.975 \\
\hline & & Nintedanib & 2 & $1008 / 1019$ & 0.0 & 0.588 & $1.188(0.832-1.696)$ & 0.0 & 1.000 & - & \\
\hline & & Sorafenib & 4 & $1010 / 935$ & 0.0 & 0.832 & $1.098(0.936-1.287)$ & 1.02 & 0.308 & 0.122 & \\
\hline & & Sunitinib & 3 & $586 / 589$ & 0.0 & 1.000 & $1.548(1.067-2.248)$ & 0.0 & 1.000 & 0.659 & \\
\hline & & Vandetanib & 9 & $2528 / 2199$ & 59.1 & 0.012 & $1.607(1.155-2.234)$ & 1.15 & 0.251 & 0.179 & \\
\hline & Line & $1 \mathrm{st}$ & 8 & $2260 / 2192$ & 0.002 & 68.6 & $1.317(1.077-1.611)$ & 0.62 & 0.536 & 0.420 & 0.345 \\
\hline & & $\geq 2$ nd & 13 & $4072 / 3717$ & 37.5 & 0.084 & $1.427(1.231-1.653)$ & 0.06 & 0.951 & 0.576 & \\
\hline & & Maintenance & 2 & $155 / 124$ & 72.6 & 0.056 & $2.254(0.321-15.819)$ & 0.00 & 1.000 & - & \\
\hline & Regimen & n Combination & 17 & $4959 / 4869$ & 50.1 & 0.010 & $1.396(1.212-1.608)$ & 0.62 & 0.537 & 0.533 & 0.129 \\
\hline & & Monotherapy & 6 & $1528 / 1164$ & 52.0 & 0.064 & $1.607(1.056-3.014)$ & 0.75 & 0.452 & 0.090 & \\
\hline \multirow[t]{8}{*}{ DCR } & TKIs & Nintedanib & 2 & $1008 / 1019$ & 61.0 & 0.109 & $1.225(1.069-1.404)$ & 0.0 & 1.000 & - & 0.938 \\
\hline & & Sorafenib & 4 & $1010 / 935$ & 75.8 & 0.006 & $1.081(0.873-1.338)$ & 1.02 & 0.308 & 0.095 & \\
\hline & & Sunitinib & 2 & $521 / 522$ & 0.0 & 0.653 & $1.209(1.052-1.389)$ & 0.0 & 1.000 & - & \\
\hline & & Vandetanib & 5 & $1700 / 1718$ & 0.0 & 0.504 & $1.137(1.056-1.224)$ & 0.0 & 1.000 & 0.065 & \\
\hline & Line & $1 \mathrm{st}$ & 4 & $1867 / 1833$ & 83.7 & 0.000 & $0.920(0.796-1.063)$ & 1.70 & 0.089 & 0.068 & 0.023 \\
\hline & & $\geq 2$ nd & 11 & $3390 / 3345$ & 19.8 & 0.255 & $1.178(1.121-1.238)$ & 1.01 & 0.312 & 0.065 & \\
\hline & Regimen & n Combination & 12 & $4501 / 4445$ & 76.0 & 0.000 & $1.089(1.004-1.182)$ & 0.34 & 0.732 & 0.765 & 0.357 \\
\hline & & Monotherapy & 3 & $756 / 733$ & 0.1 & 0.367 & $1.412(1.101-1.812)$ & 0.52 & 0.602 & - & \\
\hline
\end{tabular}

HR: hazard ratio, RR: relative risk, PFS: progression-free survival, OS: overall survival, ORR: objective response rate, DCR: disease control rate, TKIs: tyrosine kinase inhibitors. HR for PFS and OS. RR for ORR and DCR. 
significant improvement in PFS was observed in both VEGFR-TKIs monotherapies (HR:0.707, 95\%CI: 0.5600.892) and combination therapies of VEGFR-TKIs with chemotherapy (HR:0.835, 95\%CI: 0.798-0.875) (Figure $5 \mathrm{~A})$. We further performed meta-regression by the covariates including individual VEGFR-TKI, treatment line and treatment regimen. As was found in the subgroup analysis, individual VEGFR-TKI $(P=0.819)$, treatment line $(P=0.416)$ and treatment regimen $(P=0.261)$ did not result in the inter-study heterogeneity (Table 2 ).
A

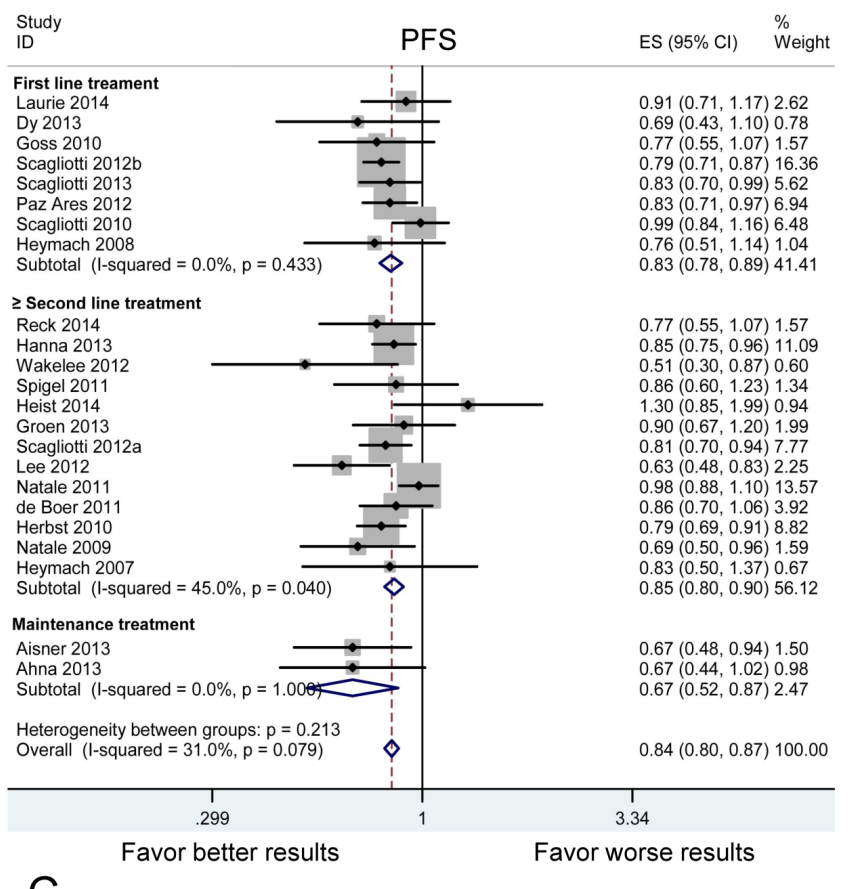

C

Stu

First line treament

Laurie 2014

Dy 2013

Scagliotti 2012b

Scagliotti 2013

Paz Ares 2012

Heymach 2008

Subtotal $($ I-squared $=68.6 \%, p=0.002)$

$\geq$ Second line treatment

Reck 2014

Hanna 2013

Wakelee 2012

Spigel 2011

Heist 2014

Groen 2013

Scagliotti 2012

Lee 2012

de Boer 2011

Herbst 2010

Natale 2009

Subtotal (l-squared $=37.5 \%, p=0.084$ )

Maintenance treatment

Aisner 2013

Ahn 2013

Subtotal ( $(-$ squared $=72.6 \%, p=0.056$ )

Overall (I-squared $=51.6 \%, p=0.002$ )

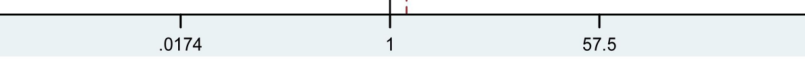

Favor worse results
ORR $\quad$ ES $(95 \% \mathrm{Cl}) \quad \stackrel{\%}{\text { Weight }}$

$1.54(1.18,2.01) \quad 8.44$ $0.92(0.38,2.23) \quad 2.11$ $\begin{array}{ll}2.38(1.50,3.77) & 5.28 \\ 1.57(1.37 .1 .79) & 10.89\end{array}$ $0.66(0.34,1.27) \quad 3.36$ $1.08(0.85,1.37) \quad 9.05$ $1.14(0.91,1.42) \quad 9.29$ $1.29(0.70,2.37) \quad 3.73$ $1.32(1.08,1.61) \quad 52.14$

$1.33(0.77,2.29) \quad 4.34$ $1.09(0.68,1.75) \quad 5.13$ $0.62(0.04,9.58) \quad 0.26$ $0.75(0.28,2.00) \quad 1.76$ $1.54(0.60,3.94) \quad 1.90$ $1.55(0.27,8.92) \quad 0.62$ $1.55(1.02,2.35) \quad 5.88$ $\begin{array}{ll}3.98(0.92,17.21) & 0.86 \\ 1.00(0.74,1.36) & 7.74\end{array}$ $\begin{array}{ll}2.42(1.51,3.88) & 5.14 \\ 1.69(1.28,2.23) & 8.23\end{array}$ $\begin{array}{ll}1.69 \\ 7.17(0.90,57.06) & 0.45\end{array}$ $1.49(0.53,4.19) \quad 1.62$ $1.45(1.16,1.81) \quad 43.94$

$1.02(0.54,1.94) \quad 3.44$ $7.84(1.07,57.49) \quad 0.48$ $2.25(0.32,15.82) 3.92$

$1.37(1.19,1.58) \quad 100.00$

Favor better results $\begin{array}{ll}1.00(0.74,1.36) & 7.74 \\ 2.42(1.51,3.88) & 5.14\end{array}$
B

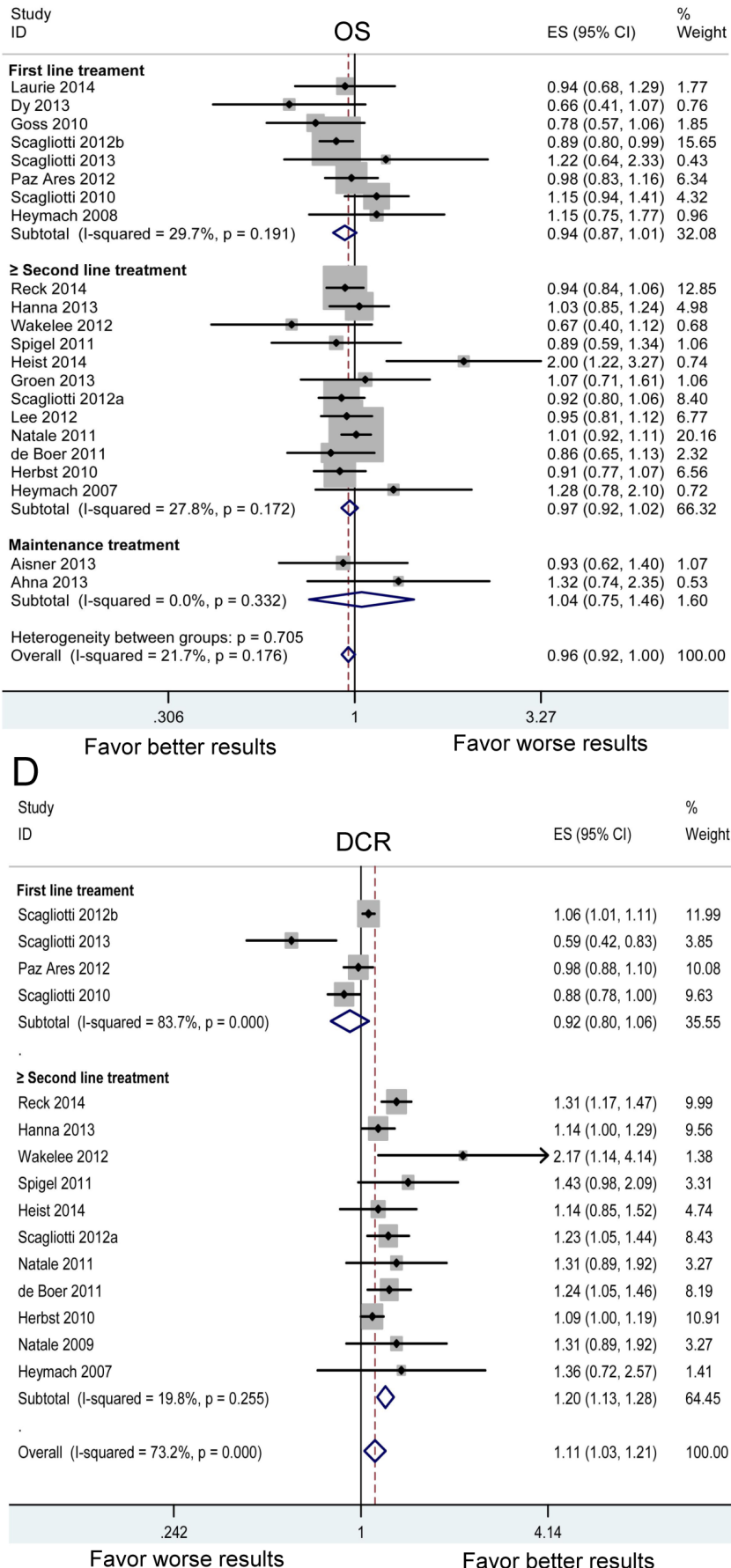

Favor better results

Figure 4: Subgroup analysis based on treatment line in advanced NSCLC patients in terms of progression-free survival (PFS) A. overall survival (OS) B. objective response rate (ORR) C. and disease control rate (DCR). D. Squares indicate study-specific HR or RR (size of the square reflects the study-specific statistical weight); horizontal lines indicate $95 \%$ confidence interval (CI); diamond indicates the summary HR or RR estimate with its $95 \% \mathrm{CI}$. 


\section{Overall survival}

The meta-analysis of OS was based on 22 RCTs provided the required data. Between-study heterogeneity could be ignored (chi-squared $=26.83$, d.f. $=21, P=$ 0.176 , I-squared $=21.7 \%$ ). There was no significant difference between VEGFR-TKIs group and control
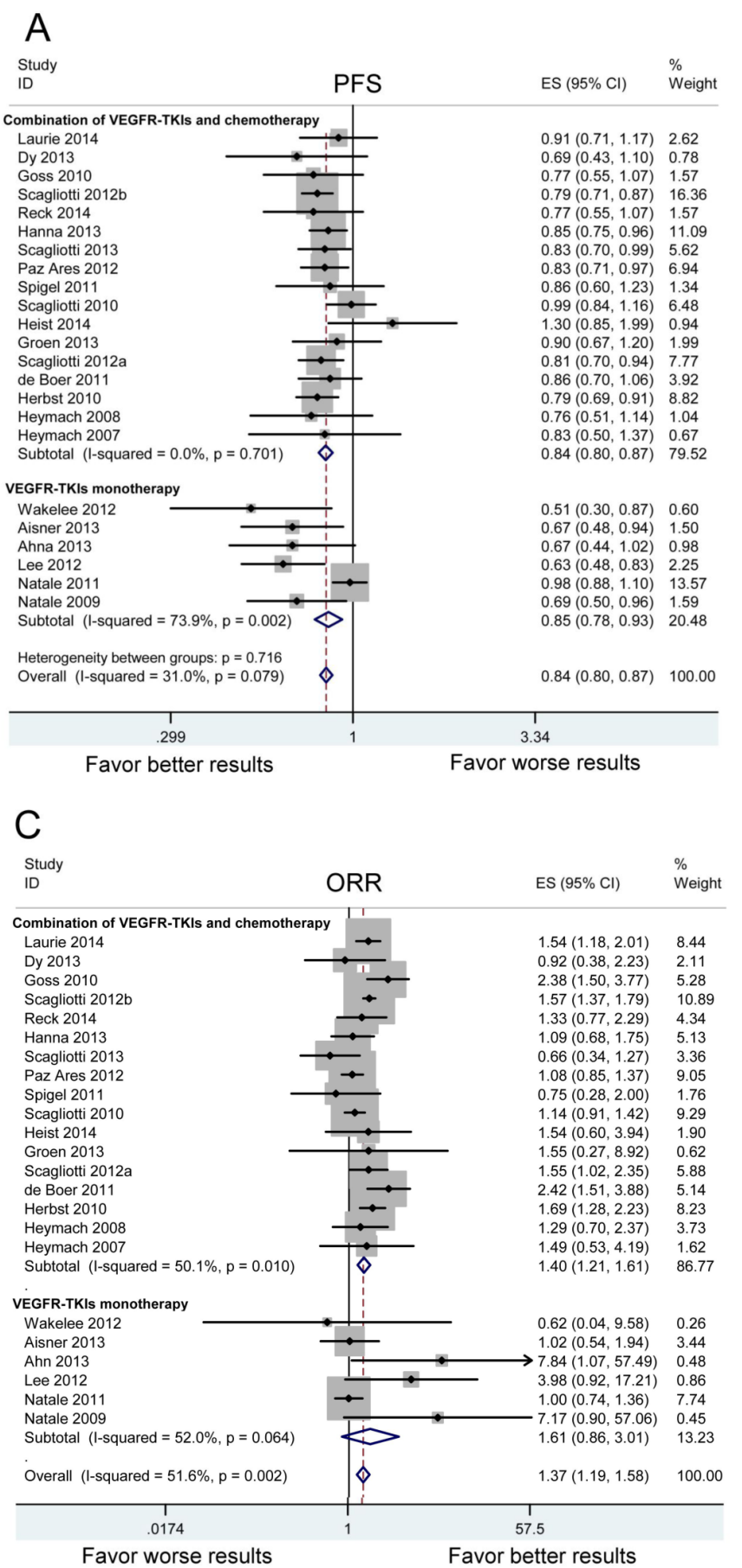

group for OS (HR:0.960, 95\%CI: 0.921-1.002, $P=$ 0.060 ) (Figure $2 \mathrm{~B}$ ). In stratified analyses by individual VEGFR-TKI, significant OS benefit was not found in cediranib, nintedanib, sorafenib, sunitinib and vandetanib (Figure 3B). A positive effect of VEGFR-TKIs for OS was not observed in first-line treatment, $\geq$ secondline treatment, and maintenance treatment (Figure

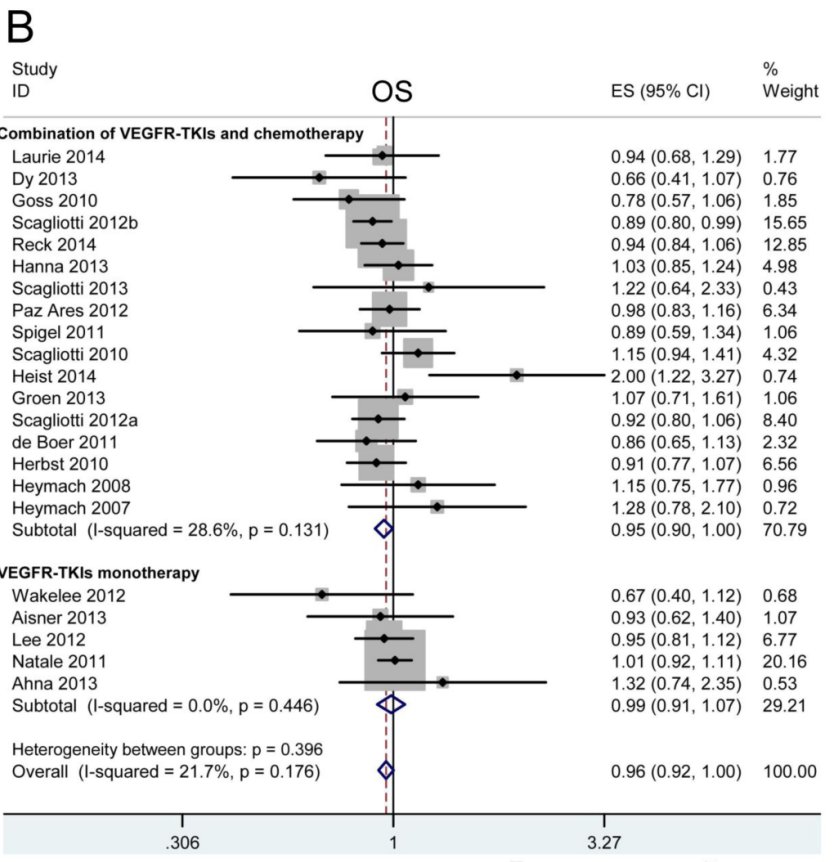

Favor better results $\quad$ Favor worse results

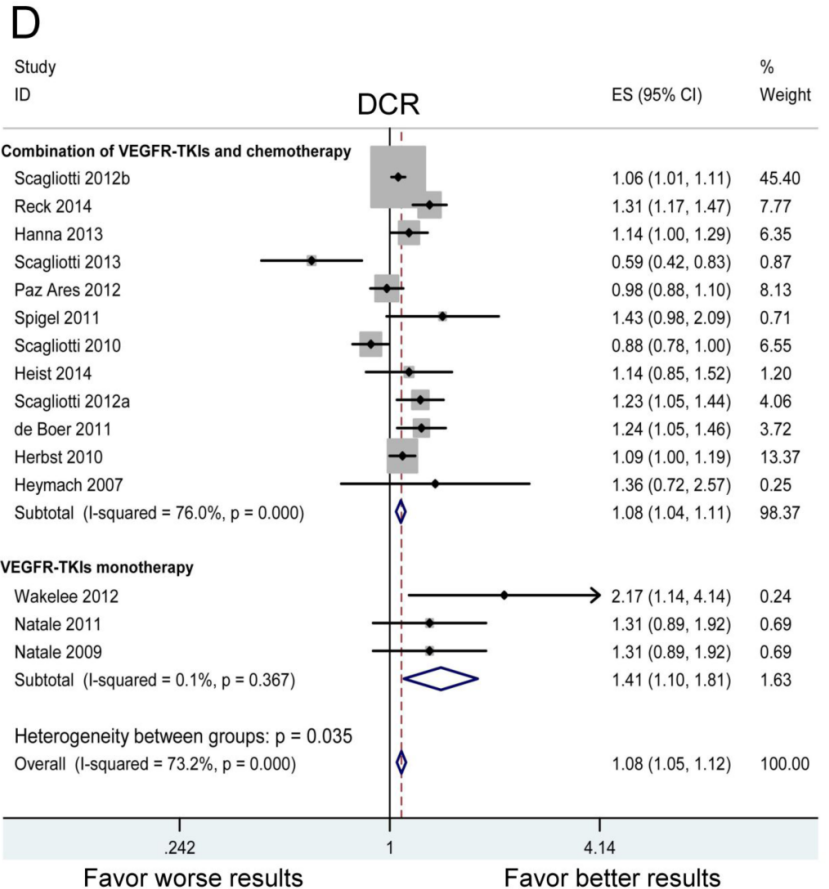

Figure 5: Subgroup analysis based on treatment regimen in advanced NSCLC patients in terms of progression-free survival (PFS) A. overall survival (OS) B. objective response rate (ORR) C. and disease control rate (DCR). D. Squares indicate study-specific HR or RR (size of the square reflects the study-specific statistical weight); horizontal lines indicate $95 \%$ confidence interval (CI); diamond indicates the summary HR or RR estimate with its $95 \% \mathrm{CI}$. 
4B). A statistically significant improvement in OS was observed in combination therapies of VEGFR-TKIs with chemotherapy, not in VEGFR-TKIs monotherapies (Figure 5B). Meta regression suggested that individual VEGFR-TKI $(P=0.322)$, treatment line $(P=0.271)$ and treatment regimen $(P=0.227)$ did not alter the pooled HR significantly (Table 2 ).

\section{Overall response rate and disease control rate}

Twenty three RCTs provided information in detail about ORR, while DCR were suggested in only fifteen trials. The results of pooled analysis showed VEGFRTKIs significantly improved ORR [relative risk (RR): 1.374, 95\%CI: 1.193-1.583, $P<0.001]$ and DCR (RR: 1.113, 95\%CI: 1.027-1.206, $P=0.009$ ) (Figure 2C, 2D).

In stratified analyses regarding individual VEGFR-

TKI, three VEGFR-TKIs (cediranib, sunitinib and vandetanib) resulted in a significant improvement of ORR (Figure 3C). Three agents (nintedanib, sunitinib and vandetanib) resulted in a significant increase of DCR (Figure 3D). The significant ORR benefit was found both in first-line and $\geq$ second-line treatment. However, better DCR was only found in $\geq$ second-line treatment (Figure 4D). Subgroup analysis showed that both monotherapy and combination therapy improved ORR and DCR (Figure 5C, 5D). Meta regression indicated that none of the examined factors were responsible for between-study heterogeneity on ORR, including individual VEGFR-TKI $(P=0.975)$, treatment line $(P=0.345)$ and treatment regimen $(P=$ $0.129)$. In addition, individual VEGFR-TKI $(P=0.938)$ and treatment regimen $(P=0.357)$ did not result in significantly heterogeneity across studies on DCR. While, treatment line $(P=0.023)$ could be a important factor responsible for between-study heterogeneity on DCR (Table 2).
A

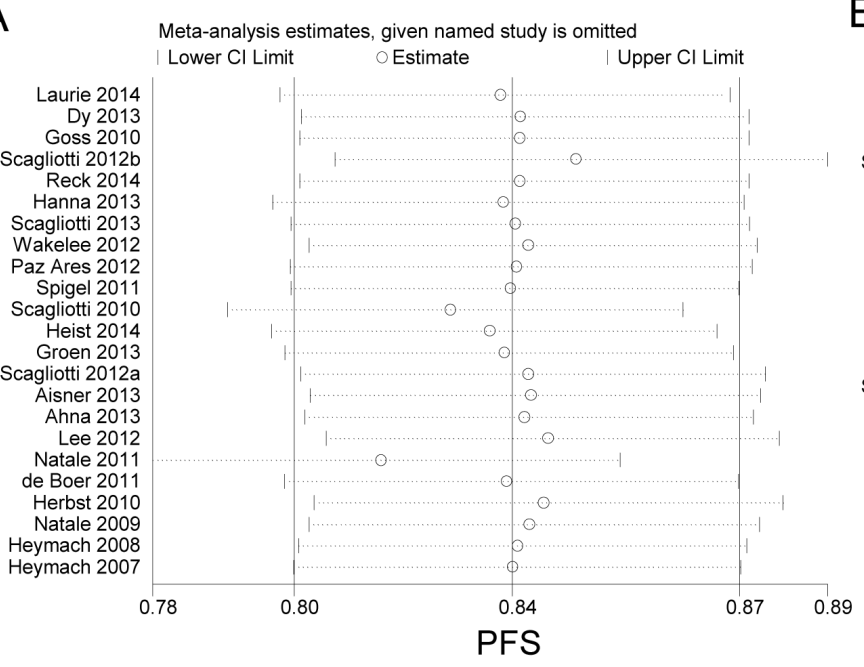

C

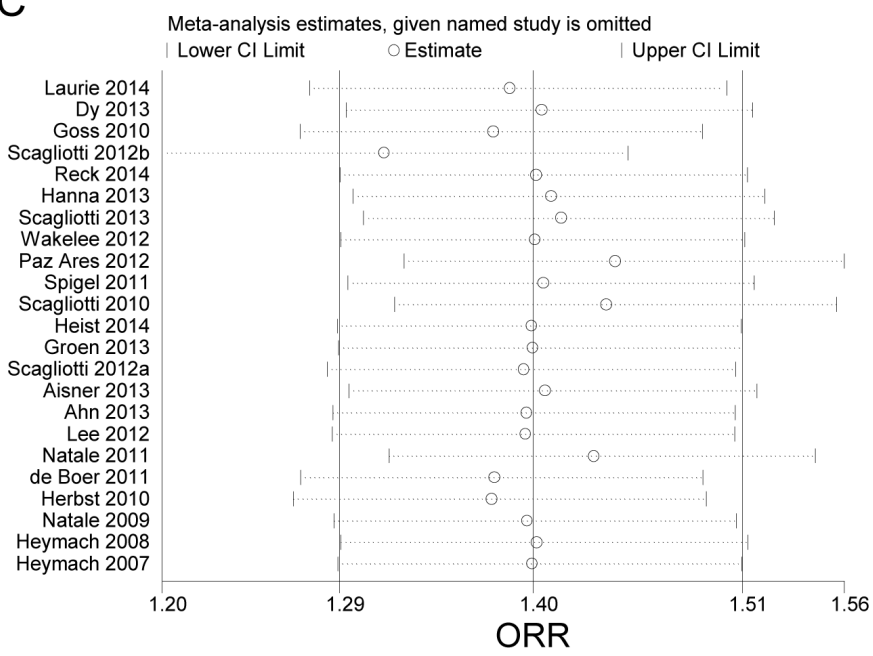

B

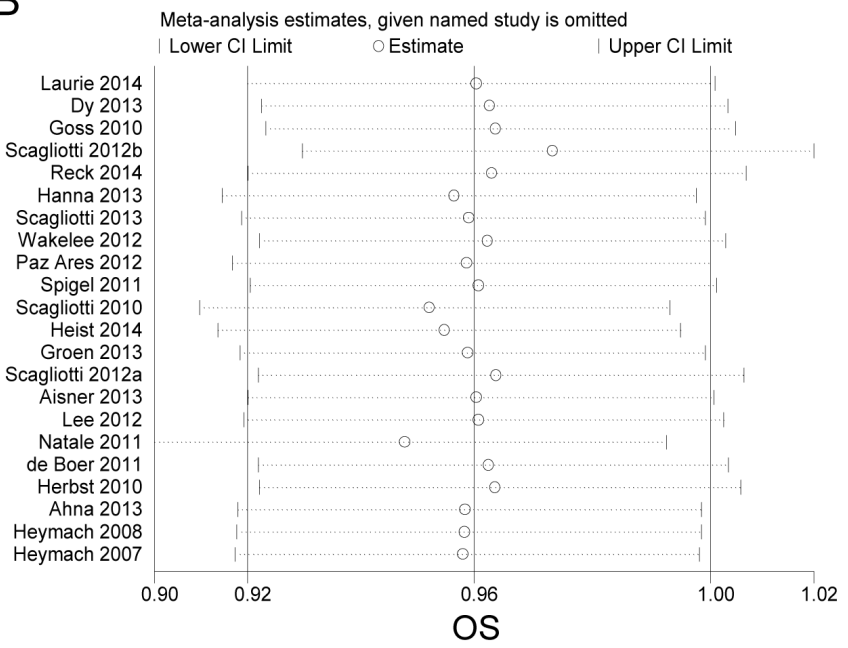

$\mathrm{D}$

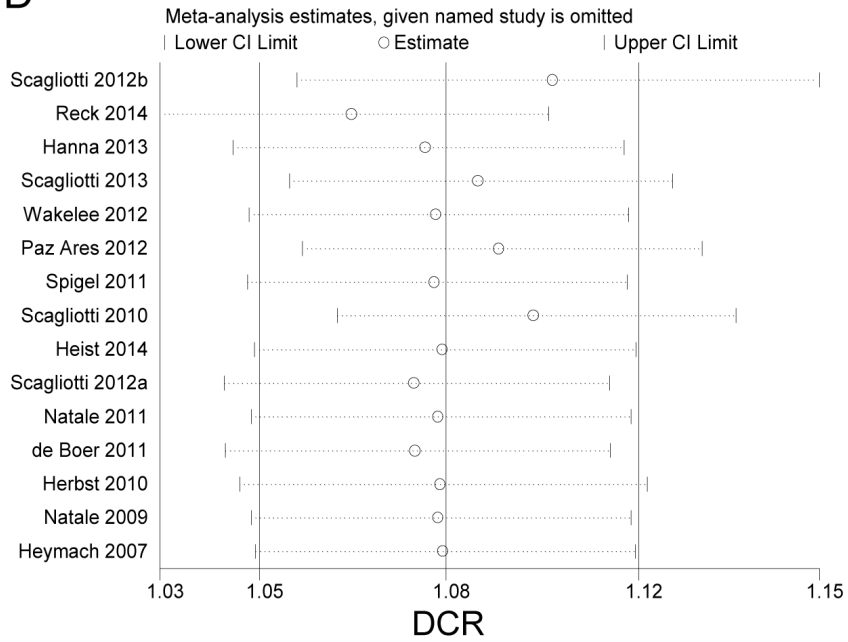

Figure 6: Sensitivity analysis of enrolled studies on progression-free survival (PFS) A. overall survival (OS) B. objective response rate (ORR) C. and disease control rate (DCR) D. 


\section{Common adverse events}

The common AEs were summarized in Table 3. The pooled analyses showed that the risks of allgrade neutropenia, thrombocytopenia, hypertension, hemorrhage, fatigue, anorexia, stomatitis, diarrhea, rash, HFSR were higher in patients receiving VEGFRTKIs. The pooled RR indicated the risks of all-grade thromboembolism, dyspnea and neuropathy were comparable between VEGFR-TKIs and control group. However, the risk of all-grade anemia was decreased in patients treated with VEGFR-TKIs than those in control group (RR:0.820, 95\%CI:0.683-0.984).

To clarified the severity of AEs, we further analyzed the $\geq 3$ grade AEs in VEGFR-TKIs and control group. Compared with the control group, the VEGFR-TKIs group showed a higher incidence of $\geq 3$ grade neutropenia, thrombocytopenia, hypertension, fatigue, stomatitis, diarrhea, rash and HFSR. Patients receiving VEGFRTKIs experienced a comparable risk of $\geq 3$ grade anemia,

A

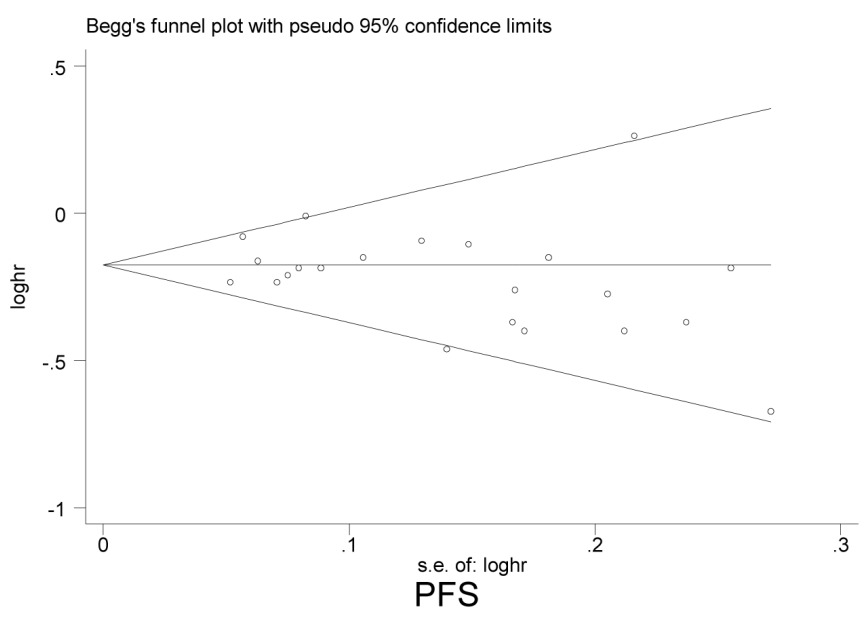

C

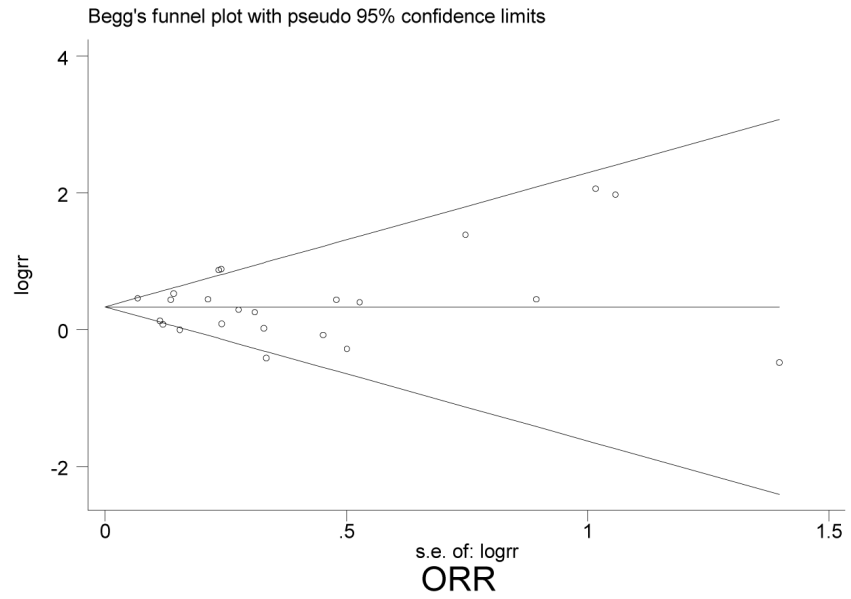

hemorrhage, thromboembolism, anorexia, dyspnea and neuropathy (Table 3).

\section{Sensitivity analyses}

We carried out sensitivity analyses to assess the stability of the results by sequentially omitting each study. The leave-one-out sensitivity analyses indicated that no individual study changed the pooled data qualitatively, suggesting that our results were stable and reliable (Figure $6)$.

\section{Publication bias}

The Begg's funnel plot and Egger's test were conducted to assess the publication bias. The shapes of the funnel plots seemed symmetrical in all meta-analyses, suggesting the absence of publication bias (Figure 7). Z-value (continuity corrected) of Begg's test in the pooled

B

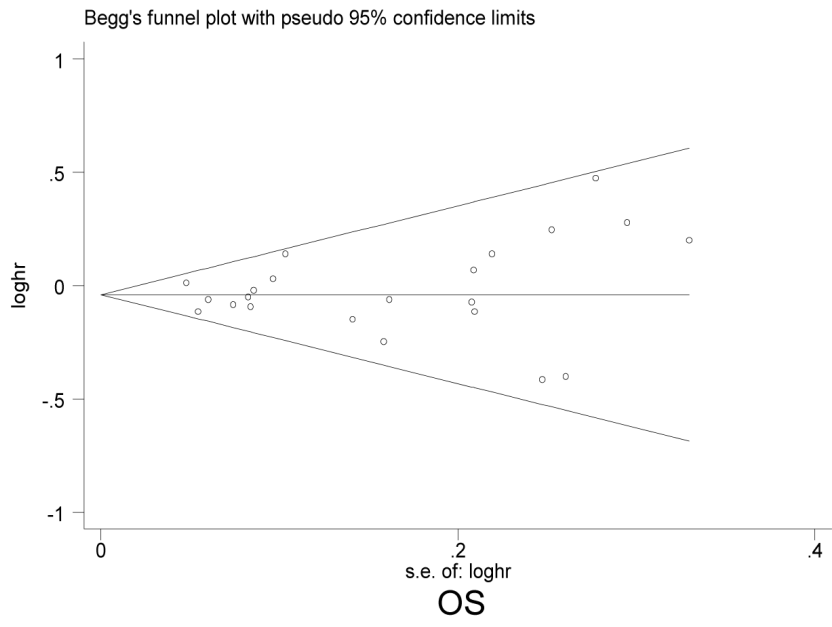

D

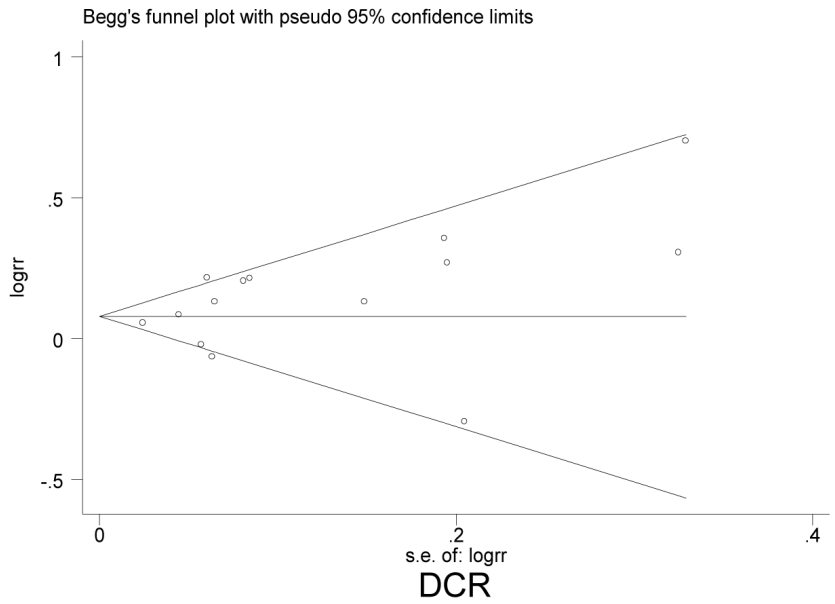

Figure 7: Begg's funnel plots of included studies on progression-free survival (PFS) A. overall survival (OS) B. objective response rate (ORR) $C$. and disease control rate (DCR) D. 
Table 3: Relative risk (RR) of common adverse events in advanced non-small cell lung cancer patients treated angiogenesis inhibitors

\begin{tabular}{|c|c|c|c|c|c|c|}
\hline \multirow[b]{2}{*}{ Adverse events } & \multicolumn{3}{|c|}{ All grades } & \multicolumn{3}{|c|}{ Grade $\geq 3$} \\
\hline & No. of studies & RR $(95 \%$ CI $)$ & $P$ value & No. of studies & RR $(95 \%$ CI $)$ & $P$ value \\
\hline Anemia & 9 & $0.820(0.683-0.984)$ & 0.033 & 12 & $0.821(0.634-1.063)$ & 0.135 \\
\hline Neutropenia & 11 & $1.445(1.091-1.914)$ & 0.010 & 11 & $1.370(1.065-1.762)$ & 0.014 \\
\hline Thrombocytopenia & 9 & $1.783(1.170-2.716)$ & 0.007 & 10 & $2.376(1.710-3.302)$ & $<0.001$ \\
\hline Hypertension & 12 & $4.012(2.951-5.455)$ & $<0.001$ & 13 & $5.693(3.668-8.834)$ & $<0.001$ \\
\hline Hemorrhage & 6 & $1.968(1.556-2.489)$ & $<0.001$ & 7 & $1.932(0.933-3.997)$ & 0.076 \\
\hline Thromboembolism & 3 & $1.074(0.644-1.792)$ & 0.784 & 6 & $0.920(0.620-1.366)$ & 0.681 \\
\hline Fatigue & 17 & $1.094(1.002-1.195)$ & 0.046 & 19 & $1.558(1.334-1.820)$ & $<0.001$ \\
\hline Anorexia & 10 & $1.209(1.030-1.420)$ & 0.020 & 12 & $1.327(0.976-1.803)$ & 0.071 \\
\hline Stomatitis & 9 & $1.896(1.377-2.611)$ & $<0.001$ & 8 & $3.835(1.721-8.546)$ & 0.001 \\
\hline Diarrhea & 17 & $1.946(1.655-2.287)$ & $<0.001$ & 20 & $2.747(1.908-3.954)$ & $<0.001$ \\
\hline Dyspnea & 10 & $0.997(0.907-1.096)$ & 0.870 & 13 & $0.873(0.729-1.045)$ & 0.139 \\
\hline Rash & 17 & $1.661(1.278-2.159)$ & $<0.001$ & 16 & $3.065(1.626-5.778)$ & 0.001 \\
\hline HFSR & 5 & $3.909(1.910-8.001)$ & $<0.001$ & 5 & $18.137(6.657-49.414)$ & $<0.001$ \\
\hline Neuropathy & 6 & $0.943(0.823-1.082)$ & 0.405 & 6 & $1.129(0.704-1.808)$ & 0.615 \\
\hline
\end{tabular}

HFSR, hand-foot skin reaction; Hemorrhage: nose, CNS, lung and abdominal hemorrhage;

analysis on PFS was $0.98(P=0.328), 1.02$ on OS $(P=$ $0.310), 0.42$ on ORR $(P=0.673)$ and 1.04 on DCR $(P$ $=0.299$ ). Egger's test showed that the t value (bias) of the meta-analyses on PFS was $-1.54(P=0.138), 0.79$ OS $(P=0.439), 0.12$ on ORR $(P=0.439)$ and 0.99 on DCR $(P=0.338)$. As shown in Table 2 , the results of Begg's test and Egger's test indicated no significant evidence for publication bias of the subgroup analyses. Bias from publications might not have a significant influence on the results of current meta-analyses. Therefore, we did not perform non-parametric "trim-and-fill" method to adjust pooled HRs or RRs.

\section{DISCUSSION}

The present report with updated data improved our understanding about the efficacy and safety of VEGFRTKIs in advanced NSCLC. The pooled results showed that VEGFR-TKIs were associated with significant improvement in PFS, ORR and DCR compared with control therapies. The basis of VEGFR-TKIs therapy is stemmed from recognition of the fact that VEGF is the most important growth factor for angiogenesis [4, 31]. VEGFR-TKIs compete with ATP for the activation domain and block intracellular VEGF signaling pathway [31].
Thus, VEGFR-TKIs result in regression of blood vessels, suppression of tumor angiogenesis and shrinkage of tumor volume.

Our results demonstrated significant association between improvement of PFS and VEGFR-TKIs (HR:0.839, 95\%CI: 0.805-0.874, $P<0.001$ ). However, the improvement of PFS with VEGFR-TKIs failed to translate into OS benefit (Figure 2). Our results were consistent with previous studies $(11,13,20-36)$. One possible explanation is that PFS is a direct indicator for treatment efficacy, while the OS may be influenced by the post-progression treatment. Patients in VEGFRTKIs group have longer PFS than control group. The improvement of symptom relief results in the possibility that patients could live with fewer symptoms for a longer time. Patients in control groups would receive more postprogression interventions. Therefore, they get important palliative benefit compared with patients in VEGFR-TKIs groups [11]. Another possible explanation is that tumor upregulates the expression of alternative pro-angiogenic factors, such as fibroblast growth factor, ephrin and angiopoietin after VEGFR-TKIs treatment [32]. Those factors compensate quickly the inhibition of VEGFR signal pathway. The exact reasons remain unclear. Thus, further studies with functional analyses are needed to 
address this issue.

In the present meta-analysis, we found the spectrum of VEGFR-TKIs associated AEs was consistent with previous studies $[11,13,33]$. Hypertension is a wellknown AE of VEGFR-TKIs. VEGFR-TKIs induce vasoconstriction by inhibition flow-mediated dilation and nitroglycerin- mediated dilation [34]. Interestingly, occurrence of treatment-related hypertension is associated with benefit of VEGFR-TKIs [35]. However, it is unclear whether hypertension might exploited as an indicator for better PFS and OS in patients treated with VEGFR-TKIs. VEGF signal pathway plays a vital role in hematopoiesis. So, VEGFR-TKIs may lead to neutropenia and thrombocytopenia. However, the novel thing is that the risk of all-grade anemia was decreased in patients treated with VEGFR-TKIs (RR:0.820, 95\%CI:0.683-0.984). One possible reason is the potential benefit of VEGFR-TKIs for reduction tumor burden. Another reason might be related to the increase of erythropoietin induced by antiangiogenic effect [36]. But the mechanism is not fully understood and further studies are needed.

In 2014, nintedanib (BIBF1120), under the brand name vargatef, was approved in European Union as a second line agent in advanced lung adenocarcinoma [17, 37]. Recent scientific evidence showed that nintedanib in combination with docetaxel prolonged survival of patients with advanced lung adenocarcinoma [37]. Nintedanib is a triple angiokinase inhibitor that simultaneously blocks VEGFR, PDGFR and fibroblast growth factor receptors proangiogenic pathways [38]. These three cellular signal pathways have an important role in angiogenesis, progression and metastasis of malignant tumors [3, 4, 39]. Several novel VEGFR-TKIs are also under clinical investigation. Angiogenesis is imperative for the tumor growth and metastasis. The formation and remodeling of vessels is mediated by stimulating molecules released from malignant cells [4, 40]. The stimulating molecules activate cellular signal pathways resulting in new vessels formation. VEGFR-TKIs inhibit sprouting of the vessels through blocking those activating signal pathways [31, 33]. Unlike classical cytotoxic drugs, VEGFR-TKIs have no direct cell-killing effect on malignant cells. Conventional chemotherapy drugs could directly kill normal and cancerous cells through inhibiting proliferation, interfering metabolism or/and inducing apoptosis. Taken together, the combination of VEGFR-TKIs and chemotherapy results in considerable malignant cells death and rapid tumor shrinkage $[32,37]$. One of the advantages is that it shows more effective anti-cancer activity than chemotherapy agent alone. Another advantage is that combination therapy could reduce the dose of cytotoxic drugs, and minimize normal cells death caused by cytotoxic drugs.

Previous studies showed that hypoxia expedited tumor invasion and metastasis by inducing hepatocyte growth factor and hypoxia-inducible factor (HIF) [41, 42]. One interesting question attracts our attention: whether
VEGFR-TKIs drive tumor progression and metastasis in hypoxia. The shifting from normoxia $\left(21 \% \mathrm{O}_{2}\right)$ to hypoxia indeed activates cancer cells for aggressive behavior [41]. However, "normoxia" defined as $21 \% \mathrm{O}_{2}(160 \mathrm{mmHg})$ is not physiological. The oxygen levels in advanced NSCLC is just about 1\% (5 mmHg) [43]. VEGFR-TKIs shift malignant cells from hypoxia (the normal oxygen level in NSCLC) to deeper hypoxia or anoxia. The metastasis and invasion of cancer is stimulated by physiological hypoxia not inflicting hypoxia [44]. VEGFR-TKIs exhaust oxygen and starve cells to death. Thus, metastasis may not occur during VEGFR-TKIs therapy [44, 45]. Effective anticancer therapy suppresses proliferation of sensitive cells and only resistant cells survive. The ideal anti-cancer therapy suppresses all malignant cells. There may be no residual cells. Currently, VEGFR-TKIs therapy is limited mainly by low efficacy and shortage of selective drugs. Blagosklonny $\mathrm{MV}$ had proposed strategies to increase efficacy: combination of VEGFR-TKIs with metronomic chemotherapy, anti-HIF drugs and inactive prodrugs [44]. Given various potential therapeutic methods, we can imagine effective VEGFR-TKIs therapies of the future.

According to our data, VEGFR-TKIs improved PFS, ORR and DCR in advanced NSCLC. Disappointedly, clinical responses to VEGFR-TKIs therapy did not translate into OS improvements. Another interesting question attracts our attention: why therapeutic responses to VEGFR-TKIs do not prolong the survival of NSCLC patients. Anti-cancer therapies kill proliferating cells and cause remission. Cancer stem cells (CSCs) persist in residual tumor and lead to relapse. All proliferating cells are descendance of the same CSCs. The life expectancy of relapsed tumor is identical to that of initial tumor. So, anti-cancer therapies extend patients' OS time between remission and relapse [46]. However, anti-cancer therapies cannot kill all proliferating cells in NSCLC. Inevitably, CSCs hierarchy shifts to the dominance of proliferating cells [47]. Anti-cancer therapies select proliferating cells with resistance-confirming mutations. Resistant proliferating cells will be attenuated by differentiation, unless proliferating cells acquire the potential for selfrenewal [47]. Once the oncogenic resistant mutations render proliferating cells drug-resistant, apoptosisreluctant and highly malignant, more aggressive relapse tumors may occur. The relapse tumor tends to be more lethal compared to initial tumor. Therefore, there is no improvement of OS. Selection for oncogenic resistance can explain the response-survival paradox. VEGFR-TKIs could improve survival of patients as long as acquired resistance is exploited.

In this meta analysis, the AEs of VEGFR-TKIs were various, because several VEGFR-TKIs are multitargeted tyrosine kinase inhibitors. Cediranib inhibits VEGFR, PDGFR, and c-kit. Sorafenib can inhibit VEGFR and PDGFR tyrosine kinase as well as the Raf kinases. Vandetanib is a potent inhibitor of RET receptor tyrosine 
kinase, VEGFR and EGFR pathways [40]. The wide clinical use of VEGFR-TKIs has raised concerns over their AEs. Thus, an emerging issue is to identify the predictor for selecting patients who benefit from VEGFRTKIs. Although several markers have been postulated, such as VEGF, MMP-9 and IL-8 [48], no biomarker has yet been used routinely in NSCLC. So, predictive markers are greatly needed to identify the subset of patients who may gain the utmost benefit from VEGFR-TKIs.

This meta analysis comprehensively analyzed data from different studies to achieve a more robust results. However, several limitations need to be addressed. First, this meta analysis was based on study-level evidence. Thus, confounding factors (demographic characteristics and post-progression treatment) could not be incorporated into analysis. An individual patient data-based metaanalysis would give more reliable results. Second, due to lack of original data, we did not perform sub-analysis based on predictive markers to identify the exact benefit population. Third. our conclusions came from the sum of 23 RCTs; 6 of which were the comparison between case and placebo; 17 of which were the comparison between TKI added on chemotherapy vs chemotherapy alone. The little heterogeneity across studies may enhance the reliability of this study. Although subgroup analyses and meta regression both demonstrated the regimen did not change the overall results significantly, inconsistent HRs and RRs for different regimen should be noticed. Therefore, further research with updated data from individual patient are needed to clarify the efficacy and safety of VEGFR-TKIs.

\section{MATERIALS AND METHODS}

\section{Search strategy and selection criteria}

Two investigators independently searched PubMed, EMBASE, Cochrane Library databases as well as Web of science to identify the articles with the following key words: NSCLC [All Fields]" or "lung cancer [All Fields]" and "cediranib (AZD2171) [All Fields]" or "motesanib (AMG706) [All Fields]" or "nintedanib (BIBF1120) [All Fields]" or "pazopanib (GW786034) [All Fields]" or "sorafenib (BAY43-9006) [All Fields]" or "sunitinib (SU11248) [All Fields]" or "vandetanib (ZD6474) [All Fields]" or "VEGFR [All Fields]" or "TKIs [All Fields]". Meeting abstracts from the American society of Clinical Oncology, World Congress of Lung Cancer and European Cancer Organization were also hand searched. We also screened the reference lists of review articles and original papers. Results were double-checked and disagreements were resolved by discussion. The published language was limited to English and literature search was conducted up to 5 December 2014.
Results from the initial search that matched the criteria below were eligible. (a) Individuals with advanced NSCLC must be histopathologically confirmed. (b) The studies must be prospective randomized controlled phase II or phase III trials on advanced NSCLC patients. (c) No patient received VEGFR-TKIs or anti-VEGFR antibodies treatment before the randomized controlled trials. (d) The studies must reported one of the four endpoints (PFS, OS, ORR or DCR). For full text review, trials were excluded if (a) clinical trials compared VEGFR-TIKs with anti-VEGF antibodies; (b) data were not available regarding primary or secondary end points; (c) the number of patients for the AEs assessment was not provided; (d) patients with small cell lung cancer or other malignancies or benign lung tumors; (e) patients were not randomized into different groups. If the same patient population was used in more than one study, only the complete study would be included.

\section{Data extraction and definition}

The Preferred Reporting Items for Systematic Reviews and Meta analyses (PRISMA) statements were used to provide complete information about this meta analysis [49]. The 27 items of PRISMA statements were shown in Supplement Checklist 1, which included the title, abstract, methods, results, discussion and funding. According to PRISMA statements, all data were independently extracted by two authors using standardised data compilation forms. The discrepancies were resolved by discussion to validate the accuracy of extraction. The primary end point was defined as PFS to standardize data collection. The secondary end points included OS, ORR, DCR as well as common AEs. The DFS was defined as the time from random assignment to disease progression. The OS time was calculated from random assignment to the date of death from any cause. Tumor response was defined as progressive disease, stable disease, partial response or complete response based on the Response Evaluation Criteria in Solid Tumors criteria [50]. The ORR was defined as the proportion of patients showing complete or partial response. The DCR included stable disease, partial response and complete response for longer than three months. Common Terminology Criteria for Adverse Events (CTCAE 3.0) was used to grade the severity of AEs. The quality of included RCTs was assessed according to Jadad scale [51].

\section{Statistical analysis}

HRs and corresponding 95\% CIs were pooled for PFS and OS. The HRs and 95\% CIs were extracted as previously reported [52-54]. The most accurate method was to obtain parameters directly from the articles or to calculate the HRs from $\mathrm{O}-\mathrm{E}$ statistic and variance. The 
second method was to estimate the HRs from sample size, survival rate at specified times, log rank statistic and $\mathrm{P}$ value. Otherwise, Kaplan-Meier Curves were analyzed using the Engauge Digitizer version 4.1 (http://digitizer. sourceforge.net/) to retrieved HRs and 95\% CIs. For measurement of RRs and their $95 \%$ CIs, we constructed $2 \times 2$ tables based on abstracted data from each RCTs. RRs and their $95 \%$ CIs were pooled to evaluate overall ORR, DCR and risk of AEs.

The Mantel-Haenszel method was used to determine the choice of the fixed effects model or the random-effects model. A sensitivity analysis was carried out by excluding each study at a time individually. The publication bias was analysed using Begg's funnel plots and Egger's linear regression test [55]. $P<0.05$ by t-test was defined as significant publication bias. Despite little heterogeneity across studies, meta regression was performed to evaluate potential effects of clinical covariables on overall outcomes. Three categorical variable were investigated, including drug class, treatment line and regimens. Univariate meta-regression analyses were carried out using the random-effects model. The restricted maximum likelihood method was undertaken to estimate the residual between-trial variance and heterogeneity degree [56]. Monte Carlo permutation test was used with 10,000 random permutations [57]. This meta-analysis was carried out using the software Stata 11.0 (Stata Corporation, College Station, TX, USA). All the P values were twosided. Differences were considered statistically significant at $P<0.05$.

\section{CONCLUSIONS}

In summary, this study provides proof of principle that VEGFR-TKIs have an advantage in terms of PFS, ORR and DCR, compared with control therapies. However, advanced NSCLC patients treated with VEGFRTKIs have high risks of AEs. Thus, the monitoring AEs during VEGFR-TKIs therapy is recommended. The risk and benefit of VEGFR-TKIs must be evaluated carefully to select patients who utmost benefit from VEGFR-TKIs treatment.

\section{ACKNOWLEDGMENTS}

We appreciated Luying Si (Ph.D, Division of Biostatistics, School of Public Health, Shandong University, Jinan, People's Republic of China) for her support on statistical data analysis.

\section{FUNDING}

This work had no specific funding.

\section{CONFLICTS OF INTERESTS}

No potential conflicts of interest were disclosed.

\section{REFERENCES}

1. Jemal A, Siegel R, Ward E, Hao Y, Xu J, Murray T, Thun MJ. Cancer statistics, 2008. CA Cancer J Clin. 2008;58:7196.

2. Schiller JH, Harrington D, Belani CP, Langer C, Sandler A, Krook J, Zhu J, Johnson DH; Eastern Cooperative Oncology Group. Comparison of four chemotherapy regimens for advanced non-small-cell lung cancer. N Engl J Med. 2002;346:92-98.

3. Parums DV. Current status of targeted therapy in non-small cell lung cancer. Drugs Today (Barc). 2014;50:503-525.

4. Zetter BR. Angiogenesis and tumor metastasis. Annu Rev Med. 1998;49:407-424.

5. de Boer RH, Arrieta Ó, Yang CH, Gottfried M, Chan V, Raats J, de Marinis F, Abratt RP, Wolf J, Blackhall FH, Langmuir P, Milenkova T, Read J, et al. Vandetanib plus pemetrexed for the second-line treatment of advanced nonsmall cell lung cancer: a randomized, double-blind phase III trial. J Clin Oncol. 2011;29:1067-1074.

6. Herbst RS, Sun Y, Eberhardt WE, Germonpré P, Saijo N, Zhou C, Wang J, Li L, Kabbinavar F, Ichinose Y, Qin $\mathrm{S}$, Zhang L, Biesma B, et al. Vandetanib plus docetaxel versus docetaxel as second-line treatment for patients with advanced non-small-cell lung cancer (ZODIAC): a doubleblind, randomised, phase 3 trial. Lancet Oncol. 2010;11:619-626.

7. Heymach JV, Paz-Ares L, De Braud F, Sebastian M, Stewart DJ, Eberhardt WE, Ranade AA, Cohen G, Trigo JM, Sandler AB, Bonomi PD, Herbst RS, Krebs AD, et al. Randomized phase II study of vandetanib alone or with paclitaxel and carboplatin as first-line treatment for advanced non-small-cell lung cancer. J Clin Oncol. 2008;26:5407-5015.

8. Heymach JV, Johnson BE, Prager D, Csada E, Roubec J, Pesek M, Spásová I, Belani CP, Bodrogi I, Gadgeel S, Kennedy SJ, Hou J, Herbst RS. Randomized, placebocontrolled phase II study of vandetanib plus docetaxel in previously treated non small-cell lung cancer. J Clin Oncol. 2007;25:4270-4277.

9. Scagliotti G, Novello S, von Pawel J, Reck M, Pereira JR, Thomas M, Abrão Miziara JE, Balint B, De Marinis F, Keller A, Arén O, Csollak M, Albert I, et al. Phase III study of carboplatin and paclitaxel alone or with sorafenib in advanced non small-cell lung cancer. J Clin Oncol. 2010;28:1835-1842.

10. Scagliotti GV, Vynnychenko I, Park K, Ichinose Y, Kubota K, Blackhall F, Pirker R, Galiulin R, Ciuleanu TE, Sydorenko O, Dediu M, Papai-Szekely Z, Banaclocha $\mathrm{NM}$, et al. International, randomized, placebo-controlled, 
double-blind phase III study of motesanib plus carboplatin/ paclitaxel in patients with advanced nonsquamous non-small-cell lung cancer: MONET1. J Clin Oncol. 2012;30:2829-2836.

11. Xiao YY, Zhan P, Yuan DM, Liu HB, Lv TF, Song Y, Shi $\mathrm{Y}$. Chemotherapy plus multitargeted antiangiogenic tyrosine kinase inhibitors or chemotherapy alone in advanced NSCLC: a meta analysis of randomized controlled trials. Eur J Clin Pharmacol. 2013;69:151-159.

12. Hong S, Fang W, Liang W, Yan Y, Zhou T, Qin T, Wu X, Ma Y, Zhao Y, Yang Y, Hu Z, Xue C, Hou X, et al. Risk of treatment-related deaths with vascular endothelial growth factor receptor tyrosine kinase inhibitors: a metaanalysis of 41 randomized controlled trials. Onco Targets Ther. 2014;7:1851-1867.

13. Hong S, Tan M, Wang S, Luo S, Chen Y, Zhang L. Efficacy and safety of angiogenesis inhibitors in advanced non-small cell lung cancer: a systematic review and meta-analysis. J Cancer Res Clin Oncol. 2014 Nov 6. DOI: 10.1007/s00432014-1862-5 [Epub ahead of print].

14. Laurie SA, Solomon BJ, Seymour L, Ellis PM, Goss GD, Shepherd FA, Boyer MJ, Arnold AM, Clingan P, Laberge F, Fenton D, Hirsh V, Zukin M, et al. Randomised, doubleblind trial of carboplatin and paclitaxel with daily oral cediranib or placebo in patients with advanced non-small cell lung cancer: NCIC Clinical Trials Group study BR29. Eur J Cancer. 2014;50:706-712.

15. Dy GK, Mandrekar SJ, Nelson GD, Meyers JP, Adjei AA, Ross HJ, Ansari RH, Lyss AP, Stella PJ, Schild SE, Molina JR, Adjei AA. A randomized phase II study of gemcitabine and carboplatin with or without cediranib as first-line therapy in advanced non-small-cell lung cancer: north Central Cancer Treatment Group Study N0528. J Thorac Oncol. 2013;8:79-88.

16. Goss GD, Arnold A, Shepherd FA, Dediu M, Ciuleanu TE, Fenton D, Zukin M, Walde D, Laberge F, Vincent MD, Ellis PM, Laurie SA, Ding K, et al. Randomized, double-blind trial of carboplatin and paclitaxel with either daily oral cediranib or placebo in advanced non-small-cell lung cancer: NCIC clinical trials group BR24 study. J Clin Oncol. 2010;28:49-55.

17. Reck M, Kaiser R, Mellemgaard A, Douillard JY4, Orlov S, Krzakowski M, von Pawel J, Gottfried M, Bondarenko I, Liao M, Gann CN, Barrueco J, Gaschler-Markefski B, et al. Docetaxel plus nintedanib versus docetaxel plus placebo in patients with previously treated non-small-cell lung cancer (LUME-Lung 1): a phase 3, double-blind, randomised controlled trial. Lancet Oncol. 2014;15:143-155.

18. Nasser H. Hanna RK, Richard N, Sullivan, Osvaldo Rudy Aren, Myung-Ju Ahn, Beatrice Tiangco, Zanete Zvirbule, Carlos H. Barrios, Ahmet Demirkazik, Birgit Gaschler-Markefski, Isabelle Voccia, et al. A multicenter, randomized, double-blind, phase III study of nintedanib plus pemetrexed versus placebo plus pemetrexed in patients with advanced nonsquamous non-small cell lung cancer
(NSCLC) after failure of first-line chemotherapy. J Clin Oncol. 31, 2013 (suppl; abstr 8034).

19. Scagliotti GV, Felip E, Besse B, von Pawel J, Mellemgaard A, Reck M, Bosquee L, Chouaid C, Lianes-Barragán P, Paul EM, Ruiz-Soto R, Sigal E, Ottesen LH, et al. An openlabel, multicenter, randomized, phase II study of pazopanib in combination with pemetrexed in first-line treatment of patients with advanced-stage non-small-cell lung cancer. J Thorac Oncol. 2013;8:1529-1537.

20. Wakelee HA, Lee JW, Hanna NH, Traynor AM, Carbone DP, Schiller JH. A double-blind randomized discontinuation phase-II study of sorafenib (BAY 43-9006) in previously treated nonsmall-cell lung cancer patients: eastern cooperative oncology group study E2501. J Thorac Oncol. 2012;7:1574-1582.

21. Paz-Ares LG, Biesma B, Heigener D, von Pawel J, Eisen T, Bennouna J, Zhang L, Liao M, Sun Y, Gans S, Syrigos K, Le Marie E, Gottfried M, et al. Phase III, randomized, double-blind, placebo- controlled trial of gemcitabine/ cisplatin alone or with sorafenib for the first-line treatment of advanced, nonsquamous non-small-cell lung cancer. J Clin Oncol. 2012;30:3084-3092.

22. Spigel DR, Burris HA 3rd, Greco FA, Shipley DL, Friedman EK, Waterhouse DM, Whorf RC, Mitchell RB, Daniel DB, Zangmeister J, Bass JD, Hainsworth JD. Randomized, double-blind, placebo-controlled, phase II trial of sorafenib and erlotinib or erlotinib alone in previously treated advanced non-small-cell lung cancer. J Clin Oncol. 2011;29:2582-2589.

23. Heist RS, Wang X, Hodgson L, Otterson GA, Stinchcombe TE, Gandhi L, Villalona-Calero MA, Watson P, Vokes EE, Socinski MA; Alliance for Clinical Trials in Oncology. CALGB 30704 (Alliance): a randomized phase II study to assess the efficacy of pemetrexed or sunitinib or pemetrexed plus sunitinib in the second-line treatment of advanced nonsmall-cell lung cancer. J Thorac Oncol. 2014;9:214-221.

24. Groen HJ, Socinski MA, Grossi F, Juhasz E, Gridelli C, Baas P, Butts CA, Chmielowska E, Usari T, Selaru P, Harmon C, Williams JA, Gao F, et al. A randomized, double-blind, phase II study of erlotinib with or without sunitinib for the second-line treatment of metastatic non-small-cell lung cancer (NSCLC). Ann Oncol. 2013;24:2382-2389.

25. Scagliotti GV, Krzakowski M, Szczesna A, Strausz J, Makhson A, Reck M, Wierzbicki RF, Albert I, Thomas M, Miziara JE, Papai ZS, Karaseva N, Thongprasert S, et al. Sunitinib plus erlotinib versus placebo plus erlotinib in patients with previously treated advanced non-small-cell lung cancer: a phase III trial. J Clin Oncol. 2012;30:20702078.

26. Aisner J, Manola JB, Dakhil SR, Stella PJ, Sovak MA, Schiller JH. Vandetanib plus chemotherapy for induction followed by vandetanib or placebo as maintenance for patients with advanced non-small-cell lung cancer: a randomized phase 2 PrECOG study (PrE0501). J Thorac 
Oncol. 2013;8:1075-1083.

27. Ahn JS, Lee KH, Sun JM, Park K, Kang ES, Cho EK, Lee DH, Kim SW, Lee GW, Kang JH, Lee JS, Lee JW, Ahn MJ. A randomized, phase II study of vandetanib maintenance for advanced or metastatic non-small-cell lung cancer following first-line platinum-doublet chemotherapy. Lung Cancer. 2013; 82:455-460.

28. Lee JS, Hirsh V, Park K, Qin S, Blajman CR, Perng RP, Chen YM, Emerson L, Langmuir P, Manegold C. Vandetanib Versus placebo in patients with advanced non-small-cell lung cancer after prior therapy with an epidermal growth factor receptor tyrosine kinase inhibitor: a randomized, double-blind phase III trial (ZEPHYR). J Clin Oncol. 2012;30:1114-1121.

29. Natale RB, Thongprasert S, Greco FA, Thomas M, Tsai CM, Sunpaweravong P, Ferry D, Mulatero C, Whorf R, Thompson J, Barlesi F, Langmuir P, Gogov S, et al. Phase III trial of vandetanib compared with erlotinib in patients with previously treated advanced non-small cell lung cancer. J Clin Oncol. 2011;29:1059-1066.

30. Natale RB, Bodkin D, Govindan R, Sleckman BG, Rizvi NA, Capó A, Germonpré P, Eberhardt WE, Stockman PK, Kennedy SJ, Ranson M. Vandetanib versus gefitinib in patients with advanced non-small-cell lung cancer: results from a twopart, double-blind, randomized phase II study. J Clin Oncol. 2009;27:2523-2529.

31. McDougall SR, Anderson AR, Chaplain MA. Mathematical modelling of dynamic adaptive tumour-induced angiogenesis: clinical implications and therapeutic targeting strategies. J Theor Biol. 2006; 241:564-589.

32. Bergers G, Hanahan D. Modes of resistance to antiangiogenic therapy. Nat Rev Cancer. 2008;8:592-603.

33. Kubota Y. Tumor angiogenesis and anti-angiogenic therapy. Keio J Med. 2012;61:47-56.

34. Steeghs N, Gelderblom H, Roodt JO, Christensen O, Rajagopalan P, Hovens M, Putter H, Rabelink TJ, de Koning E. Hypertension and rarefaction during treatment with telatinib, a small molecule angiogenesis inhibitor. Clin Cancer Res. 2008;14:3470-3476.

35. Lombardi G, Zustovich F, Farina P, Fiduccia P, Della Puppa A, Polo V, Bertorelle R, Gardiman MP, Banzato A, Ciccarino P, Denaro L, Zagonel V. Hypertension as a biomarker in patients with recurrent glioblastoma treated with antiangiogenic drugs: a single-center experience and a critical review of the literature. Anticancer Drugs. 2013;24:90-97.

36. Alexandrescu DT, McClure R, Farzanmehr H, Dasanu CA. Secondary erythrocytosis produced by the tyrosine kinase inhibitors sunitinib and sorafenib. J Clin Oncol. 2008;26:4047-4048.

37. Popat S, Mellemgaard A, Fahrbach K, Martin A, Rizzo M, Kaiser R, Griebsch I, Reck M. Nintedanib plus docetaxel as second-line therapy in patients with non-small-cell lung cancer: a network meta-analysis. Future Oncol.
2015;11:409-420.

38. Hilberg F, Roth GJ, Krssak M, Kautschitsch S, Sommergruber W, Tontsch-Grunt U, Garin-Chesa P, Bader G, Zoephel A, Quant J, Heckel A, Rettig WJ. BIBF 1120: triple angiokinase inhibitor with sustained receptor blockade and good antitumor efficacy. Cancer Res. 2008;68:47744782.

39. Bousquet C, Lamande N, Brand M, Gasc JM, Jullienne B, Faure G, Griscelli F, Opolon P, Connault E, Perricaudet M, Corvol P. Suppression of angiogenesis, tumor growth, and metastasis by adenovirus-mediated gene transfer of human angiotensinogen. Mol Ther. 2006;14:175-182.

40. Ivy SP, Wick JY, Kaufman BM. An overview of small molecule inhibitors of VEGFR signaling. Nat Rev Clin Oncol. 2009;6:569-579.

41. Pennacchietti S, Michieli P, Galluzzo M, Mazzone M, Giordano S, Comoglio PM. Hypoxia promotes invasive growth by transcriptional activation of the met protooncogene. Cancer Cell. 2003; 3, 347-361.

42. Staller P, Sulitkova J, Lisztwan J, Moch H, Oakeley EJ, Krek W. Chemokine receptor CXCR4 downregulated by von Hippel-Lindau tumour suppressor pVHL. Nature. 2003; 425, 307-311.

43. Hockel M, Vaupel P. Tumor hypoxia: definitions and current clinical, biologic, and molecular aspects. J. Natl. Cancer Inst. 2001; 93, 266-276.

44. Blagosklonny MV. Antiangiogenic therapy and tumor progression. Cancer Cell. 2004;5:13-17.

45. Kieran MW, Folkman J, Heymach J. Angiogenesis inhibitors and hypoxia. Nat Med. 2003;9, 1104-1105.

46. Kantarjian H, Talpaz M, O'Brien S, Giles F, Faderl S, Verstovsek S, Garcia-Manero G, ShanJ, Rios MB, Champlin R, de Lima M, Cortes J. Survival benefit with imatinib mesylate therapy in patients with accelerated-phase chronic myelogenous leukemia-comparison with historic experience. Cancer 2005; 103:2099-3108.

47. Blagosklonny MV. Why therapeutic response may not prolong the life of a cancer patient: selection for oncogenic resistance. Cell Cycle. 2005;4:1693-1698.

48. Hanrahan EO, Lin HY, Kim ES, Yan S, Du DZ, McKee KS, Tran HT, Lee JJ, Ryan AJ, Langmuir P, Johnson BE, Heymach JV. Distinct patterns of cytokine and angiogenic factor modulation and markers of benefit for vandetanib and/or chemotherapy in patients with non-small-cell lung cancer. J Clin Oncol. 2010;28:193-201.

49. Moher D, Liberati A, Tetzlaff J, Altman DG, Group P. Preferred reporting items for systematic reviews and meta-analyses: the PRISMA statement. J Clin Epidemiol. 2009;62:1006-1012.

50. Therasse P, Arbuck SG, Eisenhauer EA, Wanders J, Kaplan RS, Rubinstein L, Verweij J, Van Glabbeke M, van Oosterom AT, Christian MC, Gwyther SG. New guidelines to evaluate the response to treatment in solid tumors. European Organization for Research and Treatment 
of Cancer, National Cancer Institute of the United States, National Cancer Institute of Canada. J Natl Cancer Inst. 2000;92:205-216.

51. Jadad AR, Moore RA, Carroll D, Jenkinson C, Reynolds DJ, Gavaghan DJ, McQuay HJ. Assessing the quality of reports of randomized clinical trials: is blinding necessary? Control Clin Trials. 1996;17:1-12.

52. McShane LM, Altman DG, Sauerbrei W, Taube SE, Gion M, Clark GM, Statistics Subcommittee of the NCIEORTC Working Group on Cancer Diagnostics. Reporting recommendations for tumor marker prognostic studies (REMARK). J Natl Cancer Inst. 2005; 97:1180-1184.

53. Tierney JF, Stewart LA, Ghersi D, Burdett S, Sydes MR. Practical methods for incorporating summary time-to-event data into meta-analysis. Trials. 2007;8:16.

54. Wang S, Wang Z. Prognostic value of long noncoding RNA HOTAIR in digestive system malignancies. J Gastroenterol Hepatol. 2015 Mar 9. DOI:10.1111/jgh.12940. [Epub ahead of print]

55. Egger M, Davey Smith G, Schneider M, Minder C. Bias in meta-analysis detected by a simple, graphical test. BMJ. 1997; 315: 629-634.

56. Thompson SG, Higgins JP. How should meta-regression analyses be undertaken and interpreted? Stat Med. 2002;21:1559-1573.

57. Higgins JP, Thompson SG. Controlling the risk of spurious findings from meta-regression. Stat Med. 2004;23:16631682 . 